International Journal of Modern Physics B Vol. 31, No. 32 (2017) 1799001 (39 pages) (c) World Scientific Publishing Company DOI: 10.1142/S0217979217990016

\title{
Author index Volume 31
}

Abbas, G., Raza, R., Ashfaq Ahmad, M., Ajmal Khan, M., Jafar Hussain, M., Ahmad, M., Aziz, H., Ahmad, I., Batool, R., Altaf, F. \& Zhu, B., Electrochemical investigation of mixed metal oxide nanocomposite electrode for low temperature solid oxide fuel cell

Abdalla, M. S., Khalil, E. M. \& Obada, A.-S. F., Timedependent interaction between a two-level atom and a su(1,1) Lie algebra quantum system

Abdalla, S., see Batle, J.

Abdel-Rady, A. S., Hassan, S. S. A., Osman, A.-N. A. \& Salah, A., Quantum phase transition and berry phase of the Dicke model in the presence of the Stark-shift

Abhaya, S., see Panda, A.

Abushab, K. M., see Alajerami, Y. S. M.

Afaneh, F. \& SchmidtBöcking, H., Imaging of strong field dissociative single and double ionization channels of $\mathrm{N}_{2} \mathrm{O}$

Affan, H., Friedrich, R. \& Rehman, A., Study of front instability in advectionreaction-diffusion systems

Aghemenloh, E., see Onyeaju, M. C.

Ahmad, I., see Abbas, G.
B31 (2017) 1750193

B31 (2017) 1750211

B31 (2017) 1750206

B31 (2017) 1750091

B31 (2017) 1750033

B31 (2017) 1750171

B31 (2017) 1750215

B31 (2017) 1750191

B31 (2017) 1750119

B31 (2017) 1750193
Ahmad, I., see Iqbal, R.

B31 (2017) 1750263

Ahmad, I., see Khan, I.

B31 (2017) 1750148

B31 (2017) 1750118

Ahmad, I., see Rauf, A.

Ahmad, M., see Abbas, G.

Ahmed, S., Zafar, M., Shakil, M., Choudhary, M. A. \& Hashmi, M. R.-U.-R., Ab initio study of structural, electronic, and thermal properties of $P t_{1-x} P d_{x}$ alloys

Ait Taleb, Y., see El Hadfi, $\mathrm{H}$.

Ajmal Khan, M., see Abbas, $\mathrm{G}$.

Akbudak, S., see Baaziz, H.

Akhtar, A., see Golivand, M. B.

Akın, H., Phase transition and Gibbs measures of Vannimenus model on semi-infinite Cayley tree of order three

Al-Maaitah, A. F., Sandouqa, A. S., Joudeh, B. R. \& Ghassib, H. B., Thermodynamic properties of spin-polarized ${ }^{3} \mathrm{He}$ gas in the temperature range $1 \mathrm{mK}-4 \mathrm{~K}$ from the quantum second virial coefficient

Alagha, S. I., see Alajerami, Y. S. M.

Alajerami, Y. S. M., Abushab, K. M., Alagha, S. I., Mhareb, M. H. A., Saidu, A., Kodeh, F. S. \& Kh. Ramadan Physical and optical properties of sodium borate glasses

B31 (2017) 1750003

B31 (2017) 1750093

B31 (2017) 1750202

B31 (2017) 1750171 
doped with $D y^{3+}$ ions

Alam, M. A., Zilani, M. A.

K., Parvin, F. \& Hadi, M.

A., Physical properties of ternary silicide superconductors $\mathrm{Li}_{2} \mathrm{XSi}_{3}(\mathrm{X}=\mathrm{Rh}$, $O s)$ : An ab initio study

Albertini, G., see Cardone, F.

Alexandar, A., Lakshmanan, A., Sakthy Priya, S., Surendran, P. \& Rameshkumar, P., Growth, structural, optical, piezoelectric and etching analysis of $L$ lysine p-nitrophenolate monohydrate single crystals

Algin, A., Arik, M., Senay, M. \& Topcu, G., Thermostatistics of bosonic and fermionic fibonacci oscillators

Algul, G., see Enginer, Y.

Ali, M., see Faizan, M.

Ali, Z., see Iqbal, R.

Ali, Z., see Khan, I.

Altaf, F., see Abbas, G.

Altindal, Ş., see Durmuş, P.

Aly, A. H., Elsayed, H. A., Ameen, A. A. \& Mohamed, S. H., Tunable properties of onedimensional photonic crystals that incorporate a defect layer of a magnetized plasma

Aly, A. H., Nagaty, A. \& Khalifa, Z., Propagation of acoustic waves in $2 D$ periodic and quasiperiodic phononic crystals

Aly, A. H., Sabra, W. \& Elsayed, H. A., Cutoff frequency in metamaterials photonic crystals within terahertz frequencies

Amarendra, G., see Panda, A.

Ameen, A. A., see Aly, A. $\mathrm{H}$.

An, J., Chen, J., Gou, G., Chen, H. \& Wang, W., Prediction of corrosion
B31 (2017) 1750171

B31 (2017) 1750135

B31 (2017) 1750168

B31 (2017) 1750174

B31 (2017) 1650247

B31 (2017) 1750245

B31 (2017) 1750253

B31 (2017) 1750263

B31 (2017) 1750148

B31 (2017) 1750193

B31 (2017) 1750197

B31 (2017) 1750239

B31 (2017) 1750147

B31 (2017) 1750123

B31 (2017) 1750033

B31 (2017) 1750239 fatigue crack initiation behavior of A7N01P-T4 aluminum alloy welded joints

An, N., Liu, C.-Z., Fan, C.B., Dong, X. \& Song, Q.L., Theory study on the bandgap of antimonidebased multi-element alloys

Anitha, A. \& Arulmozhi, M., Simultaneous effects of pressure and temperature on excitons in pöschlteller quantum well

Ansari, R., Rouhi, S. \& Nikkar, A., Finite element investigation of the vibrational behavior of concentric multi-walled boron nitride and carbon nanotubes

Ansari, R., see Rouhi, S.

Ao, P., Topological quantum numbers in nonrelativistic physics

Apollaro, T. J. G., see Paganelli, S.

Aporoski, A. V., see Sadykov, N. R.

Arafin, S. \& Singh, R. N., Pressure variation of melting temperatures of alkali halides

Arik, M., see Algin, A.

Ariyanti, D., Maillot, M. \& Gao, W., $\mathrm{TiO}_{2}$ used as photocatalyst for rhodamine $B$ degradation under solar radiation

Ariyanti, D., see Dong, J.

Arulmozhi, M., see Anitha, A.

Asger Mortensen, N., see Tserkezis, C.

Ashfaq Ahmad, M., see Abbas, $\mathrm{G}$.

Ata, E., Demirhan, D. \& Büyükkılıç, F., Relativistic energy-dispersion relations of $2 D$ rectang $u$ lar lattices

Audouard, A., see Gasparov, V. A.

Aziz, H., see Abbas, G.

\section{B31 (2017) 1744034}

B31 (2017) 1750089

B31 (2017) 1750050

B31 (2017) 1750018

B31 (2017) 1750262

B31 (2017) 1780001

B31 (2017) 1750065

B31 (2017) 1750017

B31 (2017) 1750031

B31 (2017) 1650247

B31 (2017) 1744095

B31 (2017) 1744049

B31 (2017) 1750050

B31 (2017) 1740005

B31 (2017) 1750193

B31 (2017) 1750061

B31 (2017) 1745016

B31 (2017) 1750193 
Baaziz, H., Dj. Guendouz., Charifi, Z., Akbudak, S., Uğur, G., Ş. Uğur \& Boudiaf, K., Investigation of the structural, electronic, elastic and thermodynamic properties of curium monopnictides: An ab initio study

Babaeipour, M., see Javaheri, S.

Babaeipour, M., see Javaheri, $\mathrm{S}$.

Bagarello, F. \& Fring, A., From pseudo-bosons to pseudo-Hermiticity via multiple generalized Bogoliubov transformations

Bahari, A., Ramzannezhad, A., Shajari, D. \& Najafi, H., Nanostructural and electrical properties of $\mathrm{LA}_{0.5} \mathrm{SR}_{0.5} \mathrm{CoO}_{3}$

Bai, Y., Zhang, Q., Zheng, F., Yang, Y., Meng, Q., Zhu, L. \& Wang, B., First-principles study on codoping effect to enhance photocatalytic activity of anatase $\mathrm{TiO}_{2}$

Bai, Y., see Liu, S.

Bai, Z.-W. \& Ding, L.P., Surface diffusion of a brownian particle subjected to an external harmonic noise

Baig, M. W., On the theory of time dilation in chemical kinetics

Bailin, H., In memoriam of professor kerson huang

Bamaarouf, O., Ould Baba, A., Lamzabi, S., Rachadi, A. \& Ez-Zahraouy, H., Effects of maximum node degree on computer virus spreading in scale-free networks

Bandyopadhyay, M. \& Jayannavar, A. M., Brownian motion of classical spins: Anomalous dissipation and generalized langevin equation

Bandyopadhyay, S., see Sad- hukhan, B.

B31 (2017) 1750218

Banerjee, K., Sharma, C. \& Bittu, N., Plunges in the Bombay stock exchange: Characteristics and indicators

Bao, K., Chen, T., Wang, X., Song, A. \& Wan, L., Bandgaps characteristics of the periodical slit metal tubes with backstraps

Bao, K., see Wang, F.

Bao, Y., see Yang, K.

Baral, P. C., Study of frequency- and temperature-dependent electrical transport in heavy fermion systems

B31 (2017) 1750085

Bassani, D., see Cardone, F.

Bastone, E. C., see Morais, C. W.

B31 (2017) 1750015

Batle, J., Tarawneh, O., Nagata, K., Nakamura, T., Abdalla, S. \& Farouk, A., Multipartite quantum correlations in the extended $J_{1}-J_{2}$ heisenberg model

Batool, R., see Abbas, G.

Bayati, P., see Rashidian, Z.

B31 (2017) 1750036 B31 (2017) 1650254

B31 (2017) 1750082

B31 (2017) 1750177

B31 (2017) 1777002

B31 (2017) 1750182

B31 (2017) 1750189
Belkacemi, G. \& Bourahla, B., Heat transfer by metallic thin film sandwiched in semiconductor lattice

Belogorlov, A. A., see Borman, V. D.

Belyaev, M., see Velichko, A.

Ben-Bolie, G. H., see Ngounga Makoundit, G. J.

Benmakhlouf, A., Errandonea, D., Bouhemadou, A., Bentabet, A., Maabed, S., Bouchenafa, M. \& Bin-Omran, S., Ab initio and electronic properties of scheelite-type $\mathrm{XWO}_{4}(\mathrm{X}$ $=\mathrm{Ca}, \mathrm{Sr}, \mathrm{Ba}$ ) compounds

Bentabet, A., see Benmakhlouf, A.

Benyoussef, A., see Houmad, M.

Benyoussef, A., see Jabar, study of the mechanical
B31 (2017) 1750160

B31 (2017) 1750125

B31 (2017) 1750049

B31 (2017) 1744030

B31 (2017) 1750081

B31 (2017) 1750168

B31 (2017) 1750237

B31 (2017) 1750206

B31 (2017) 1750193

B31 (2017) 1750043

B31 (2017) 1750155

B31 (2017) 1750201

B31 (2017) 1650261

B31 (2017) 1750150

B31 (2017) 1750086

B31 (2017) 1750086

B31 (2017) 1750163 
A.

Benyoussef, A., see Jabar, A.

Benzidi, H., see Houmad, M.

Bharadwaj, S., see Chaitanya Varma, M.

Bhattacherjee, A. B. \& Sharma, D., Enhanced spin squeezing and quantum entanglement near the critical point of the Jaynes-Cummings-Dicke model

Bi, L., see Zhang, J.

Bian, M., see Chen, Y.

Bin-Omran, S., see Benmakhlouf, A.

Bittu, N., see Banerjee, K.

Blackmore, D., Rosato, A., Sen, S. \& Wu, H., Simulation, modeling and dynamical analysis of multibody flows

Bo, H., Hou, F., Tay, S. L., Wang, Y., Gao, W. \& Xiong, C., Microstructure and properties of $\mathrm{TiO}_{2}$ sol-enhanced black nickel nanocomposite coating

Bo, L., see Guo, Q.

Bogolubov, N. N. \& Soldatov, A. V., Algebraic aspects of the driven dynamics in the density operator and correlation functions calculation for multi-level open quantum systems

Bollinger, A. T., see Božović, I.

Boochani, A., see Golivand, M. B.

Boochani, A., see Javaheri, $\mathrm{S}$.

Boochani, A., see Javaheri, $\mathrm{S}$.

Boochani, A., see Khosravi, $\mathrm{H}$.

Bordbar, G. H., Hosseini, S. \& Poostforush, A., Isentropic calculation for thermodynamic properties of polarized liquid ${ }^{3} \mathrm{He}$ by considering the
B31 (2017) 1750208

B31 (2017) 1750035

B31 (2017) 1750163

B31 (2017) 1750063

B31 (2017) 1750062

B31 (2017) 1741013

B31 (2017) 1744057

B31 (2017) 1750086

B31 (2017) 1750160

B31 (2017) 1742004

B31 (2017) 1744023

B31 (2017) 1745001

B31 (2017) 1850044

B31 (2017) 1745005

B31 (2017) 1750003

B31 (2017) 1650255

B31 (2017) 1750261

B31 (2017) 1750109 effect of spin-dependent correlation function

Borman, V. D., Borodulya, N. A., Belogorlov, A. A. \& Tronin, V. N., The self-consistent model of the anomalously slow relaxation of the systems nonwetting liquidnanoporous medium

Borodulya, N. A., see Borman, V. D.

Bouarissa, N., Boucenna, M., Saib, S. \& Siddiqui, S. A., Energy gaps, effective masses and ionicity of $A l_{x} G a_{1-x} S b$ ternary semiconductor alloys

Boucenna, M., see Bouarissa, N.

Bouchenafa, M., see Benmakhlouf, A.

Boudiaf, K., see Baaziz, H.

Boudjemâa, A., Manysoliton bound states in dispersion-managed optical fiber: Possibility of fiber-optic transmission of three bits per clock period

Bouhemadou, A., see Benmakhlouf, A.

Bourahla, B., see Belkacemi, $\mathrm{G}$.

Bozovic, I., see Gasparov, V. A.

Božović, I., He, X., Wu, J. \& Bollinger, A. T., What is strange about hightemperature superconductivity in cuprates?

$\mathrm{Bu}, \mathrm{W}$., see $\mathrm{Xu}, \mathrm{L}$.

$\mathrm{Bu}, \mathrm{Z}$., see Zhang, F.

Bulus, I., Dalhatu, S. A., Hussin, R., Wan Shamsuri, W. N. \& Yamusa, Y. A., The role of dysprosium ions on the physical and optical properties of lithiumborosulfophosphate glasses

Buryakov, A. M., see Khusyainov, D. I.

Butenko, D., see Tomchuk, P. M.
B31 (2017) 1750097

B31 (2017) 1750201

B31 (2017) 1750201

B31 (2017) 1750232

B31 (2017) 1750232

B31 (2017) 1750086

B31 (2017) 1750226

B31 (2017) 1750178

B31 (2017) 1750086

B31 (2017) 1750155

B31 (2017) 1745016

B31 (2017) 1745005

B31 (2017) 1750167

B31 (2017) 1750223

B31 (2017) 1750101

B31 (2017) 1750195

B31 (2017) 1750029 
Büyükkılıç, F., see Ata, E.

Cai, J. \& Lv, J., Defects detecting method of lamp cap of single soldering lug

Cai, J., see Hu, F.

Cai, L. L., Tian, Y. L., Yuan, X. B., Hu, G. C. \& Ren, J. F., Spin polarization properties at the spinterface of thiophene/fe(100): First principles calculations

Cai, W.-M., see Huang, X.

Cai, Z. B., see Peng, J. F.

Calcina-Nogales, M., see Mamani, E.

Cambui, D. S. \& Iliass, T., Behavioral transitions induced by speed and noise in animal aggregates

Cao, J., see Jiao, M.

Cao, J., see Yang, K.

Cao, S., see Guo, L.

Cao, W., see Chen, Y.

Cao, X., Li, X., Chen, X., $\mathrm{Xu}$, S., Xiong, D. \& Deng, W., Preparation, characterization and optical properties of Dydoped yttrium aluminum garnet

Cao, X., see Deng, W.

Cardone, F., Albertini, G., Bassani, D., Cherubini, G., Guerriero, E., Mignani, R., Monti, M., Petrucci, A., Ridolfi, F., Rosada, A., Rosetto, F., Sala, V., Santoro, E. \& Spera, G., Deformed space-time transformations in mercury

Carretero-González, R., see Kevrekidis, P. G.

Castro, J. A., see Durante Rincón, C. A

Chae, K. S., see Tegafaw, T.

Chai, J., see Xiao, J.

Chai, J.-M., see Chen, L.

Chaitanya Varma, M., Bharadwaj, S., Choudary, K., G. S. V. R., Murthy, K. S. R. \& Rao, K. H., Influence of magnesium-
B31 (2017) 1750061

B31 (2017) 1744078 B31 (2017) 1750121

B31 (2017) 1750072

B31 (2017) 1750078

B31 (2017) 1744020

B31 (2017) 1750116

B31 (2017) 1750073

B31 (2017) 1744090

B31 (2017) 1744102

B31 (2017) 1750252

B31 (2017) 1744057

B31 (2017) 1744071

B31 (2017) 1744068

B31 (2017) 1750168

B31 (2017) 1742013

B31 (2017) 1750246

B31 (2017) 1750014

B31 (2017) 1744062

B31 (2017) 1744063 substituted $\mathrm{Ni}-\mathrm{Zn}$ ferrites on magnetic and electric losses at lower frequency

Chakraborty, A., Jong, C.S., Ganguli, N. \& Dasgupta, I., A comparative study of magnetic and optical properties of $M n-$, Gd-, and Nd-doped $\mathrm{ZnO}$ nanowires

Chang, C.-H. \& Ortix, C., Ballistic anisotropic magnetoresistance in coreshell nanowires and rolled-up nanotubes

Chang, Y., see Tegafaw, T.

Chao, X., see Tay, S. L.

Charifi, Z., see Baaziz, $\mathrm{H}$.

Chase, M., see Kenkre, V. M.

Che, X., see Liu, Y.

Che, X., see Shen, L.

Chegel, R., Feyzi, A. \& Moradian, R., Electrical and optical conductivities of bilayer silicene: Tightbinding calculations

Chen, B., Zhang, Y., Ouyang, Q., Chen, F., Zhan, X. \& Gao, W., The SiNx films process research by plasmaenhanced chemical vapor deposition in crystalline silicon solar cells

Chen, B., see Chen, X.

Chen, C., see Chen, R.

Chen, D. \& Yuan, Y. J., Formaldehyde adsorption on carbon nanotubes fragment by density functional theory

Chen, F., Zhan, X., Salcic, Z., Wong, C. C. \& Gao, W., Silicon-based hot electron emitting substrate with double tunneling

Chen, F., see Chen, B.

Chen, F., see Hu, Y.

Chen, F., see Ji, L.-B.

Chen, F., see Zhan, X.

Chen, G., see Li, X.-X.

Chen, H., see An, J.

Chen, H., see Chen, W.
B31 (2017) 1744052

B31 (2017) 1750063

B31 (2017) 1650241

B31 (2017) 1630016

B31 (2017) 1750014

B31 (2017) 1744028

B31 (2017) 1750226

B31 (2017) 1744041

B31 (2017) 1744009

B31 (2017) 1750158

B31 (2017) 1744101

B31 (2017) 1750146

B31 (2017) 1750259

B31 (2017) 1744074

B31 (2017) 1744101

B31 (2017) 1750181

B31 (2017) 1744017

B31 (2017) 1744051

B31 (2017) 1750256

B31 (2017) 1744034

B31 (2017) 1744031
B31 (2017) 1750244 
Chen, H., see Chen, Y. Chen, H., see Han, D. Chen, H., see He, Y. Chen, H., see Liu, Y. Chen, H., see Liu, Y. Chen, H., see Shen, L. Chen, H., see Wang, Q. Chen, H., see Xiao, F. Chen, H., see Xi, N. Chen, H., see Zhang, K. Chen, H., see Zhang, M. Chen, H., see Zhang, X. Chen, J., see An, J.

Chen, J., see Chen, Q.-M. Chen, J., see Liu, Y.

Chen, J., see Li, H.-J.

Chen, J., see Shang, Y.-Q.

Chen, J., see Shao, Z.

Chen, J., see Yang, T.

Chen, J., see Ye, Y.

Chen, J., see Zhang, X.

Chen, L. J., see Liu, C. G.

Chen, L., Wang, Z.-Y., Hui, W., Chu, C.-Y., Chai, J.-M., Xiao, J., Zhao, Y. \& Ma, J.-X., Quantum ratchet effect in a time non-uniform doublekicked model

Chen, L., see Hu, F.

Chen, L., see Jiang, D.

Chen, L., see Qi, Y.

Chen, L., see Xiao, J.

Chen, L., see Xiong, C.

Chen, L., see Zhao, Y.

Chen, L., see Zhou, Y.

Chen, M., see Lee, W.

Chen, P., see Hu, Y.

Chen, Q.-M., Zhou, F.-F., Yuan, H.-C., Chen, J., Ni, Y., Zhu, X.-F. \& Dou, X.-M., The effect of processing on the properties of $\mathrm{CuInS}_{2}$ nanomaterials synthesized by simple hot injection route

Chen, R., Chen, C. \& Nie, L., Absolute negative mobility in the anomalous diffusion

Chen, S., see Pan, Y.

Chen, T. H., see Yan, J. G.

Chen, T., see Bao, K.

Chen, T., see Song, A.

Chen, T., see Wang, F.

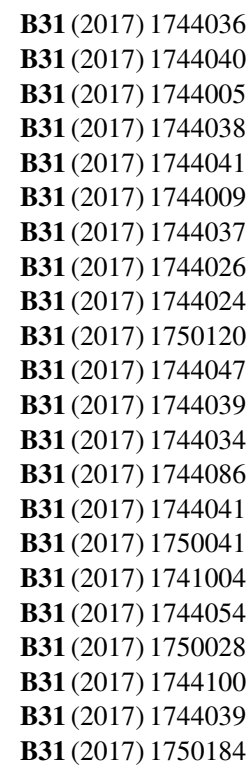

B31 (2017) 1744063

B31 (2017) 1750121

B31 (2017) 1650264

B31 (2017) 1745022

B31 (2017) 1744062

B31 (2017) 1744076

B31 (2017) 1744098

B31 (2017) 1744088

B31 (2017) 1750039

B31 (2017) 1750181

B31 (2017) 1744086

B31 (2017) 1750259

B31 (2017) 1750096

B31 (2017) 1650263

B31 (2017) 1750125

B31 (2017) 1750038

B31 (2017) 1750049
Chen, W., Chen, H., Wang, Y., Li, C. \& Wang, X., Characteristics of $\mathrm{Ni}-\mathrm{Cr}$ $\mathrm{Fe}$ laser clad layers on EA4T steel

Chen, W., Yang, T. \& Yang, R., 3d model of filler melting with micro-beam plasma arc based on additive manufacturing technology

Chen, W., see Wang, Y.

Chen, X. \& Yang, P., Synthesis and characterization of antimony-doped n-type silicon quantum dots

Chen, X., Shi, Z., Chen, B. \& Song, K., Robust and controllable electronic local transports in armchair silicene nanoribbons under a perpendicular electric field

Chen, X., see Cao, X.

Chen, X., see Cheng, X.

Chen, X., see Chen, $Z$.

Chen, Y., Chen, H., Zhu, M., Yang, T. \& Shen, L., Elements loss analysis based on spectral diagnosis in laser-arc hybrid welding of aluminum alloy

Chen, Y., Li, Z., Su, H., Xue, S., Bian, M., Xu, L., Cao, W. \& Huang, $\mathrm{Z}$., Influence of Ti content and sintering temperature on dielectric properties of $\mathrm{Bi}_{4} \mathrm{Ti}_{3} \mathrm{O}_{12}$ ceramics

Chen, Y., see Jiang, D.

Chen, Y., see Meng, M.

Chen, Y., see Sun, S. P.

Chen, Y., see Wang, Q.

Chen, Y.-C., Zhou, Y.-L. \& Wang, C., Monte Carlo simulation on the diffusion of polymer in narrow periodical channels

Chen, Z. J., see Yan, J. G.

Chen, Z., Chen, X., Li, F., Xu, S., Huang, W. \& Deng, W., Influence of $\mathrm{TiO}_{2}$ content on microstructures and optoelectronic properties
B31 (2017) 1744031

B31 (2017) 1744016 B31 (2017) 1744022

B31 (2017) 1750110

B31 (2017) 1750146

B31 (2017) 1744071

B31 (2017) 1744061

B31 (2017) 1744069

B31 (2017) 1744036

B31 (2017) 1744057

B31 (2017) 1650264

B31 (2017) 1744008

B31 (2017) 1744081

B31 (2017) 1750057

B31 (2017) 1750144 B31 (2017) 1650263 
of titanium-doped $\mathrm{ZnO}$ nanofilms

Chen, Z., see Deng, W.

Cheng, M.-T., see Ma, X. S. Cheng, Q., see Li, H.-J.

Cheng, X., Cui, H., Li, X. \& Deng, W., Investigation of ptcr effect and microdefects in $\mathrm{Nb}_{2} \mathrm{O}_{5}$ doped $\mathrm{BaTiO}_{3}$-based ceramics by positron annihilation techniques

Cheng, X., Li, X., Chen, $\mathrm{X}$. \& Cui, H., Influence of sintering process on electrical properties and ptcr effect of laminated $\mathrm{Ba}_{1.005}\left(\mathrm{Ti}_{1-x} \mathrm{Nb}_{x}\right) \mathrm{O}_{3} \mathrm{ce}$ ramics fired in a reducing atmosphere

Cheng, X., see Zhang, Y.

Cheng, Y. H., see Li, Y. F.

Chern, C.-H., see Huang, B.J.

Chern, C.-H., see Huang, B.J.

Cherubini, G., see Cardone, F.

Chi, Z., Guo, X. \& Wang, Z., Topological phase transition of two-dimensional topological polaritons

Choudary, K., G. S. V. R., see Chaitanya Varma, M.

Choudhary, M. A., see Ahmed, S.

Christov, I. C., Kress, T. \& Saxena, A., Peakompactons: Peaked compact nonlinear waves

Chu, C.-Y., see Chen, L.

Chuong, T. V., see Tuan, D. A.

Chávez, I., García, L. A., de Llano, M. \& Grether, M., Bcs-Bose crossover theory extended with hole Cooper pairs

Chávez, I., García, L. A., de Llano, M. \& Grether, M., Role of superconducting energy gap in extended BCS-Bose crossover theory

Collis, W. J. M. F., New
B31 (2017) 1744069

B31 (2017) 1744068

B31 (2017) 1650260

B31 (2017) 1750041

B31 (2017) 1744060

B31 (2017) 1744061

B31 (2017) 1750216

B31 (2017) 1750161

B31 (2017) 1750126

B31 (2017) 1750130

B31 (2017) 1750168

B31 (2017) 1750070

B31 (2017) 1750063

B31 (2017) 1650243

B31 (2017) 1742008

B31 (2017) 1744063

B31 (2017) 1650258

B31 (2017) 1745013

B31 (2017) 1745004 global atomic mass formulas

B31 (2017) 1745024

Cong, S., see Sun, Z.

Coste, C., Jean, M. S. \& Dessup, T., Stability, diffusion and interactions of nonlinear excitations in a many body system

Cottam, M. G., see Komorowski, P. G.

Criss, E. M. \& Hofmeister, A. M., Isolating lattice from electronic contributions in thermal transport measurements of metals and alloys above ambient temperature and an adiabatic model

Cuansing, E. C., Attenuation and amplification of the transient current in nanojunctions with timevarying gate potentials

Cui, H., see Cheng, X.

Cui, H., see Cheng, X.

Cui, Y., see Wang, Q.

da Silva, L. B., see de Figueiredo, J. E.

Dai, B., Zhu, J., Liu, K., Yang, L. \& Han, J., The dependence of critical current density of GdFeCo layer on composition of thermally assisted STTRAM

Dai, B., see Wang, P.

Dai, G., Shi, W., Jiang, X., Shi, G. \& Zhang, Y., Study on the antiseepage mechanism of the PBFC slurry for landfill site

Dai, J., see Zheng, H.

Dai, M.-L., see Zheng, B.$\mathrm{X}$.

Dakir, O., see Houmad, M.

Dalhatu, S. A., see Bulus, I.

Daneshmand, R., see Rustaee, $\mathrm{N}$.

Daoud, M., see El Hadfi, H.

Daoud, M., see Mansour, M.

Darvish, G., see Tamandani, S.

Das, D., see Pan, S.

Dasgupta, I., see Chakraborty, A.

\author{
B31 (2017) 1750240
}

B31 (2017) 1742003

B31 (2017) 1750220

B31 (2017) 1750205

B31 (2017) 1750105

B31 (2017) 1744060

B31 (2017) 1744061

B31 (2017) 1744037

B31 (2017) 1750157

B31 (2017) 1744072

B31 (2017) 1744087

B31 (2017) 1744066

B31 (2017) 1775001

B31 (2017) 1750163

B31 (2017) 1750101

B31 (2017) 1750006

B31 (2017) 1750052

B31 (2017) 1750132

B31 (2017) 1650253

B31 (2017) 1750019

B31 (2017) 1650241
B31 (2017) 1744075 
Datta, A., see Kashyap, R. de Figueiredo, J. E. \& da Silva, L. B., Electronic structure of POPC phospholipids under constant electric field

De Freitas, D. N., see Morais, C. W.

de Llano, M., see Chávez, I. de Llano, M., see Chávez, I. De Meglio, N., see Westley, A.

Deepa, B. \& Philominathan, P., Optical, mechanical and thermal behaviors of nitrilotriacetic acid single crystal

Dekeyser, C., see Khusyainov, D. I.

Demirhan, D., see Ata, E.

Deng, B., see Li, H.

Deng, B., see Shan, B.

Deng, B., see Su, F.

Deng, B.-L., see Shi, S.-P.

Deng, W., Chen, Z., Meng, Y., Cao, X., Xiong, D. \& Huang, Y., Micro-defects and optical properties of YAG:Ce crystals prepared by optical floating zone method

Deng, W., see Cao, X.

Deng, W., see Cheng, X.

Deng, W., see Chen, Z.

Deng, W., see Wan, Y.

Deng, Z. G., see Lei, W. Y.

Deng, Z. G., see Sun, R. X.

Deng, Z., see Zhang, J.

Dessup, T., see Coste, C.

Dey, S., Fring, A. \& Hussin, V., Nonclassicality versus entanglement in a noncommutative space

Dezashibi, F. M., see Tajik, F.

Dharamvir, K., see Dhiman, S.

Dhiman, S., Kumar, R. \& Dharamvir, K., Small Al and $\mathrm{Ga}$ clusters trapped inside the Bucky-ball $\left(c_{60}\right)$ - a DFT study

Diao, Q., see Jiao, M.

Diao, Y., Liu, L., Xia, S. \& Kong, Y., Differences
B31 (2017) 1742014

B31 (2017) 1750157

B31 (2017) 1750237

B31 (2017) 1745004

B31 (2017) 1745013

B31 (2017) 1742002

B31 (2017) 1750200

B31 (2017) 1750195

B31 (2017) 1750061

B31 (2017) 1750187

B31 (2017) 1750023

B31 (2017) 1750238

B31 (2017) 1750022

B31 (2017) 1744068

B31 (2017) 1744071

B31 (2017) 1744060

B31 (2017) 1744069

B31 (2017) 1744070

B31 (2017) 1745021

B31 (2017) 1745014

B31 (2017) 1745020

B31 (2017) 1742003

B31 (2017) 1650248

B31 (2017) 1750258

B31 (2017) 1750092

B31 (2017) 1750092 B31 (2017) 1744090 in optoelectronic properties between h-saturated and unsaturated GaN nanowires with DFT method

Die, D., see Zheng, B.-X.

Ding, H. \& Zhang, J., Superconducting phases in a generalized hubbard chain with additional offdiagonal hopping processes

Ding, L.-P., see Bai, Z.-W.

Ding, M., Zhang, W., Xie, Z., Lei, R., Wang, J. \& Gao, W., Selective separation and recovery of silver and copper from mixtures by photocatalysis

Ding, Q., Zhang, A., Zhao, G., Peng, H. \& Gao, W., Effects of $\mathrm{La}_{2} \mathrm{O}_{3}$ on the microstructure and tribological properties of plasma-sprayed $\mathrm{Cr}_{2} \mathrm{O}_{3}-$ $\mathrm{TiO}_{2}$ coatings

Ding, X., see Tao, Y.

Dioury, F., see Dufour, J.

Diwaker \& Kumar, A., A DFT study of structural and electronic properties of $Z n_{1-x} S b_{x} T e$ with $x=$ $(0.25,0.50,0.75)$

Dixit, A., Tiwari, S. \& Pandey, P. C., Optical properties of $3 r d$ order kerr hexagonal nonlinear photonic crystal fiber containing metal

Dj. Guendouz, see Baaziz, $\mathrm{H}$.

Djoufack, Z. I., KenfackJiotsa, A. \& Nguenang, J.-P., Quantum signature of breathers in $1 D$ ultracold bosons in optical lattices involving next-nearest neighbor interactions

Dolatyari, M., see Jafari, H.

Dong, H. M., Jin, Q. \& Wang, X. F., Tunable energy gaps and midinfrared optical properties in InAs/GaSb type-II
B31 (2017) 1750084

B31 (2017) 1775001

B31 (2017) 1750058

B31 (2017) 1750082

B31 (2017) 1744096

B31 (2017) 1744033

B31 (2017) 1750179

B31 (2017) 1745007

B31 (2017) 1650249

B31 (2017) 1750047

B31 (2017) 1750226

B31 (2017) 1750140 B31 (2017) 1750002 
superlattices

Dong, H., see Su, F.

Dong, J., Ariyanti, D., Gao, W., Niu, Z. \& Weil, E., Patterned titania nanostructures produced by electrochemical anodization of titanium sheet

Dong, J., see Fu, D.

Dong, J., see Zhang, Y.

Dong, L. Y., see Liu, C. G.

Dong, L., see Ren, X.

Dong, S. J., see Guan, X.

Dong, X., see An, N.

Dou, X., see Liu, C.

Dou, X.-M., see Chen, Q.M.

Drigo, L., see Gasparov, V. A.

Du, H., see Du, L.

Du, J., see Xiao, F.

Du, L., Zhang, P., Wang, L. \& Du, H., Effect of different re site ionic radii on the electronic structures and elastic properties of $\mathrm{Ba}_{2} \mathrm{RENbO}_{6}: \mathrm{A}$ first-principles study

Du, W., Yang, J., Xiong, C., Zhao, Y. \& Zhu, X., Preferential orientation growth of ITO thin film on quartz substrate with $\mathrm{ZnO}$ buffer layer by magnetron sputtering technique

Du, W., see Xiao, J.

Du, W., see Xiong, C.

Du, W.-H., see Yang, J.-J.

Du, W.-H., see Zhao, Y.

$\mathrm{Du}, \mathrm{X}$., see Tu, C.

Du, X., see Zhang, F.

Duan, J., see Zheng, H.

Duan, X. Y., see Qin, X. M.

Dufour, J., Dufour, X., Dioury, F. \& Vinko, J. D., Measurement of the enthalpy of formation of an iron pico-hydride and of its main properties

Dufour, X., see Dufour, J.

Durante Rincón, C. A., Durán, L. T., Medina, J. E., Castro, J. A., León, M. \& Fermín, J. R., Struc-
B31 (2017) 1750098

B31 (2017) 1750027

B31 (2017) 1744049

B31 (2017) 1750172

B31 (2017) 1744003

B31 (2017) 1750184

B31 (2017) 1741003

B31 (2017) 1744019

B31 (2017) 1750089

B31 (2017) 1744094

B31 (2017) 1744086

B31 (2017) 1745016

B31 (2017) 1750165

B31 (2017) 1744026

B31 (2017) 1750165

B31 (2017) 1744065

B31 (2017) 1744062

B31 (2017) 1744076

B31 (2017) 1744056

B31 (2017) 1744098

B31 (2017) 1650262

B31 (2017) 1750223

B31 (2017) 1744066

B31 (2017) 1744012

B31 (2017) 1745007

B31 (2017) 1745007 tural and optical properties of $\mathrm{AgIn}_{5} \mathrm{~S}_{8}$

Durmuş, P. \& Ş. Altindal Two-diode behavior in metal-ferroelectricsemiconductor structures with bismuth titanate interfacial layer

Durán, L. T., see Durante Rincón, C. A.

Eglitis, R. I., see Sokolov, M.

Eissa, A. M., see Ghali, M.

Ekogo, T. B., see Ngounga Makoundit, G. J.

El Hadfi, H., Ait Taleb, Y. \& Daoud, M., Quantum interferometric power and the role of nonclassical correlations in quantum metrology for a special class of two-qubit states

El Kenz, A., see Houmad, M.

Elbistan, M., Weyl semimetal and topological numbers

Elsayed, H. A., see Aly, A. H.

Elsayed, H. A., see Aly, A. $\mathrm{H}$.

Enginer, Y., Algul, G. \& Yurtseven, H., Calculation of the $P-T$ phase diagram of nitrogen using $a$ mean field model

Errandonea, D., see Benmakhlouf, A.

Esmailpour, M., Mohammadpour, H. \& Hadavifar, H., The hartman effect in graphene systems

Esposito, S., see Mauro, M. D.

Eyni, Z. \& Mohammadpour, H., A spin current rectifier

Ez-Zahraouy, H., see Bamaarouf, $\mathrm{O}$.

Ez-Zahraouy, H., see Lazfi, S.

Fai, L. C., see Lionel, T. K.

Faizan, M., Ullah, H., Khan, S. H., Ramay, S. M., Qaid, S. A. S., Mahmood, A. \& Ali, M., Carrier effective
B31 (2017) 1750246

B31 (2017) 1750197

B31 (2017) 1750246

B31 (2017) 1750251

B31 (2017) 1750037

B31 (2017) 1750150

B31 (2017) 1750052

B31 (2017) 1750163

B31 (2017) 1750221

B31 (2017) 1750123

B31 (2017) 1750239

B31 (2017) 1750245

B31 (2017) 1750086

B31 (2017) 1650250

B31 (2017) 1750213

B31 (2017) 1750230

B31 (2017) 1750182

B31 (2017) 1750228

B31 (2017) 1750046 
masses and thermoelectric properties of novel $\mathrm{Ag}_{3} \mathrm{AuSe} \mathrm{e}_{2}$ and $\mathrm{Ag}_{3} \mathrm{AuTe}_{2}$ compounds

Fan, C.-B., see An, N.

Fan, G., see Liu, P.

Fan, J. D., see Guo, Q.

Fan, J. D., see Wang, J. T.

Fan, J., see Zhao, W.

Fan, K., LWangZhu, Y., Liu, Z. \& Yu, B., Performance of a multipurpose piezoelectric energy harvester

Fan, K., Tang, J., Yang, L., $\mathrm{Hu}$, Y., Sun, Q. \& Zu, X., Dft study of hydrogen and helium defects at the (1121) twin boundary in hcp scandium

Fan, K., Yu, B., Zhu, Y., Liu, Z. \& Wang, L., Scavenging energy from the motion of human lower limbs via a piezoelectric energy harvester

Farouk, A., see Batle, J.

Fazeli, M., Sedighy, S. H. \& Hassani, H. R., Homogeneous near-perfect invisible ground and free space cloak

Feali, M. S., see Yarmohammadi, $M$.

Feng, L. \& Liu, H., Highintensity attosecond $X$ ray source generation by using low-intensity spatial inhomogeneous midinfrared pulse combined with an ultraviolet pulse

Feng, L., Li, W. \& Liu, H., Intensity enhancement of attosecond XUV pulse by using asymmetric inhomogeneous mid-infrared down-chirped field

Feng, S.-M., see Zhang, J.

Feng, T., see Zhang, Y.

Feng, X., see Xu, L. F.

Feng, Y.-H., see Wang, Y.C.

Fermín, J. R., see Durante Rincón, C. A.
B31 (2017) 1741011

B31 (2017) 1744077

B31 (2017) 1750173

B31 (2017) 1750206

B31 (2017) 1750253

B31 (2017) 1750089

B31 (2017) 1741002

B31 (2017) 1745001

B31 (2017) 1745023

B31 (2017) 1750212

B31 (2017) 1741007

B31 (2017) 1750080

B31 (2017) 1750059

B31 (2017) 1750053

B31 (2017) 1650239

B31 (2017) 1750185

B31 (2017) 1745020

B31 (2017) 1744003

B31 (2017) 1750133

B31 (2017) 1744067

B31 (2017) 1750246
Ferri, G. L., Pennini, F., Plastino, A. \& Rocca, M. C., New mathematics for the nonadditive Tsallis, scenario

Feyzi, A., see Chegel, R.

Fisher, M. P. A., Are we quantum computers, or merely clever robots?

Fortes, M., see Salas, P.

Fouokeng, G. C., see Lionel, T. K.

Frantzeskakis, D. J., see Kevrekidis, P. G.

Friedrich, R., see Affan, $\mathrm{H}$.

Fring, A., see Bagarello, F.

Fring, A., see Dey, S.

Fu, D., Zhou, H., Wang, K., Zhang, P., Dong, J., Gao, H. \& Li, F., Detecting the topological charge of a vortex beam by an arc slit diffraction

Fu, Q., see $\mathrm{Xu}, \mathrm{G}$.

Fu, Y., see Zhuo, F.

Fu, Z. H., Gou, G. Q., Xiao, J., Qiu, S. Y. \& Wang, W. J., The effect of temperature on the SCC behavior of AISI301L stainless steel welded joints in $3.5 \% \quad \mathrm{NaCl}$ solution

Furuta, T., Katou, K., Itoh, S., Tachibana, K., Ishikawa, S. \& Katsuragi, H., Geometry-dependent constitutive law for granular slow frictional drag

Galam, S., The trump phenomenon: An explanation from sociophysics

B31 (2017) 1750151

B31 (2017) 1750158

B31 (2017) 1743001

B31 (2017) 1750100

B31 (2017) 1750046

B31 (2017) 1742013

B31 (2017) 1750191

B31 (2017) 1750085

B31 (2017) 1650248

B31 (2017) 1750172

B31 (2017) 1750034

B31 (2017) 1750076

B31 (2017) 1744048

B31 (2017) 1742006

B31 (2017) 1742015

Galbova, O., Peschansky, V. G. \& Stepanenko, D. I., Magnetotransport phenomena in layered conductors under magnetic breakdown

Galiev, G. B., see Khusyainov, D. I.

Ganguli, N., see Chakraborty, A.

Gao, H., see Fu, D.

Gao, J., see Mamat, A.

Gao, J., see Qi, Y.
B31 (2017) 1750114

B31 (2017) 1750195

B31 (2017) 1650241

B31 (2017) 1750172

B31 (2017) 1745002

B31 (2017) 1745022 
Author index

Gao, J., see Zhu, X.

Gao, N., Wu, J. H., Hou, H. \& Yu, L., Excellent lowfrequency sound absorption of radial membrane acoustic metamaterial

Gao, W., Wang, G., Li, X., Hu, X. \& Song, H., Improved thermoelectric properties of holedoped $\mathrm{Bi}_{2-x} \mathrm{Na}_{x} \mathrm{Ba}_{2} \mathrm{Co}_{2} \mathrm{O}_{y}$ ceramics

Gao, W., see Ariyanti, D.

Gao, W., see Bo, H.

Gao, W., see Chen, B.

Gao, W., see Chen, F.

Gao, W., see Ding, M.

Gao, W., see Ding, Q.

Gao, W., see Dong, J.

Gao, W., see Hou, F.

Gao, W., see Hou, H.

Gao, W., see Jia, P.

Gao, W., see Liu, Y.

Gao, W., see Tay, S. L.

Gao, W., see Wang, Y.

Gao, W., see Wang, Y.

Gao, W., see Wang, Y.

Gao, W., see Xi, N.

Gao, W., see Yao, C.

Gao, W., see Yao, C.

Gao, W., see Zhang, Y.

Gao, W., see Zhan, X.

Gao, X., see Peng, T.

Gao, Y. M., see Li, Y. F.

Gao, Y., Wang, J. \& Ma, F., Studies on the signal amplification in weighted and unweighted smallworld networks

Gao, Y., see Sun, L.

García de Abajo, F. J., see Trügler, A.

García, L. A., see Chávez, I.

García, L. A., see Chávez, I.

Gasparov, V. A., Audouard, A., Drigo, L., He, X. \& Bozovic, I., Study of high- $T_{c}$ interface superconductivity in $\mathrm{La}_{1.55} \mathrm{Sr}_{0.45} \mathrm{CuO}_{4} / \mathrm{La}_{2} \mathrm{CuO}$ heterostructures at high magnetic fields and frequencies

Geneste, J.-F. \& Vinko, J. D., Towards a physical lattice
B31 (2017) 1745008

B31 (2017) 1750011

B31 (2017) 1750042

B31 (2017) 1744095

B31 (2017) 1744023

B31 (2017) 1744101

B31 (2017) 1744052

B31 (2017) 1744096

B31 (2017) 1744033

B31 (2017) 1744049

B31 (2017) 1744029

B31 (2017) 1744002

B31 (2017) 1744099

B31 (2017) 1744050

B31 (2017) 1744028

B31 (2017) 1744001

B31 (2017) 1744022

B31 (2017) 1744025

B31 (2017) 1744024

B31 (2017) 1744004

B31 (2017) 1744082

B31 (2017) 1744003

B31 (2017) 1744051

B31 (2017) 1750016

B31 (2017) 1750161

B31 (2017) 1750021

B31 (2017) 1750012

B31 (2017) 1740007

B31 (2017) 1745004

B31 (2017) 1745013

B31 (2017) 1745016 model for the atom and implications for high-Tc superconductivity

Gerhardt, I., Grotz, B., Siyushev, P. \& Wrachtrup, J., Coherent interaction of single molecules and plasmonic nanowires

Gh. Khorrami, H., Mousavi, M., Khayatian, S. A., Kompany, A. \& Khorsand Zak, A., Green synthesis and characterization of $\mathrm{ANbO}_{3} \quad(\mathrm{~A}=$ $\mathrm{Na}, \mathrm{K})$ nanopowders fabricated using a biopolymer

Ghali, M., Eissa, A. M. \& Mosaad, M. M., Crystalline phase transformation of colloidal cadmium sulfide nanocrystals

Ghassib, H. B., see AlMaaitah, A. F.

Ghobadi, Z. K., Shokri, A. \& Zarei, S., Effect of boron impurity in a carbon nanotube superlattice

Ghoreishi, S. S. \& Yousefi, R., A computational study of a novel graphene nanoribbon field effect transistor

Ghosh, S. \& Muley, A., Acousto-electric interaction in inhomogeneous semiconductor quantum plasma

Giri, R., see Mishra, A. C.

Gochitashvili, M. R., see Lomsadze, R. A.

Golivand, M. B., Boochani, A., Akhtar, A., Torkashvand, M. \& Karimian, N., $A b$ initio study of optical and vibrational properties of $\mathrm{Ni}_{3} \mathrm{C}$

Golshan, M. M., see Saadi, M. F.

Gong, P., see Li, S.-L.

Gong, T., see Li, J.

Gord, J. R., see Roy, S.

Gou, G. Q., see Fu, Z. H.

Gou, G., see An, J.

Gou, G., see Liu, Y.
B31 (2017) 1745009

B31 (2017) 1740004

B31 (2017) 1750194

B31 (2017) 1750037

B31 (2017) 1750202

B31 (2017) 1750106

B31 (2017) 1750056

B31 (2017) 1750207

B31 (2017) 1750162

B31 (2017) 1650257

B31 (2017) 1750003

B31 (2017) 1750001

B31 (2017) 1750173

B31 (2017) 1745006

B31 (2017) 1742007

B31 (2017) 1744048

B31 (2017) 1744034

B31 (2017) 1744041 
Gou, G., see Zhang, M.

Goudarzi, H., see Roshanzamir-Nikou, M.

Govindaraj, R., see Panda, A.

Grether, M., see Chávez, I.

Grether, M., see Chávez, I.

Grotz, B., see Gerhardt, I.

Gu, D., see Zhang, F.

Gu, H., see Tao, Y.

Gu, Q., see Zhao, J.

Gu, X., see Jiang, D.

Guan, W., see Huang, A.

Guan, X., Luo, P. \& Dong, S. J., Hydrogen production using aluminum-based materials prepared by mechanical milling

Guerriero, E., see Cardone, F.

Gul, M., see Rauf, A.

Gunaratne, G. H., see Roy, $\mathrm{S}$.

Guo, H., see Huang, X.

Guo, K., see Hu, M.

Guo, L., Cao, S. \& Wang, L., Electron beam irradiation of fluorinated graphene

Guo, L., see Xu, G.

Guo, L., see Xu, G.

Guo, Q., Zhang, P., Bo, L., Zeng, G., Li, D., Fan, J. D. \& Liu, H., An application of high-temperature superconductors YBCO to magnetic separation

Guo, S., Peng, Y., Han, X. \& Li, J., Frequency dependence of dielectric characteristics of seawater ionic solution under static magnetic field

Guo, S., see Wang, P.

Guo, W., see Lv, B.

Guo, X., see Chi, Z.

Guo, Y. X., see Yang, W. M.

Guo, Y., see Liao, L.

Guo, Y., see Song, J.

Guo, Y., see Zhang, C.

Guo, Y.-F., Xi, B., Wei, F. \& Tan, J.-G., Stochastic resonance in FitzhughNagumo neural system driven by correlated nongaussian noise and gaus-
B31 (2017) 1744047

B31 (2017) 1750013

B31 (2017) 1750033

B31 (2017) 1745004

B31 (2017) 1745013

B31 (2017) 1740004

B31 (2017) 1750223

B31 (2017) 1750179

B31 (2017) 1745011

B31 (2017) 1650264

B31 (2017) 1750087

B31 (2017) 1744019

B31 (2017) 1750168

B31 (2017) 1750118

B31 (2017) 1742007

B31 (2017) 1750078

B31 (2017) 1750009

B31 (2017) 1750252

B31 (2017) 1750030

B31 (2017) 1750034

B31 (2017) 1745001

B31 (2017) 1750169

B31 (2017) 1744072

B31 (2017) 1745017

B31 (2017) 1750070

B31 (2017) 1745018

B31 (2017) 1750067

B31 (2017) 1750222

B31 (2017) 1750079 sian noise

Guo, Z.-N., see Guo, Z.-Z.

Guo, Z.-Z. \& Guo, Z.-N., The inverse spin hall effect in the lateral spin valve structure with dual injectors

Gupta, A. S., see Pan, S.

Gökçek, N., see Kandemir, B. S.

Ha, N. T. T., Yen, N. V., Hung, P. K. \& Hong, N. V., The dynamics and structure heterogeneity of aluminum-silicate melts: Molecular dynamics simulation

Ha, V. T. T., Hung, V. V., Hanh, P. T. M., Nguyen, V. T. \& Hieu, H. K., Structural and thermomechanical properties of the zinc-blende $A l X(X=\mathrm{P}$, $A s, S b)$ compounds

Hadavifar, H., see Esmailpour, $\mathrm{M}$.

Hadi, M. A., see Alam, M. A.

Hager, R., see Westley, A.

Hamedoun, M., see Jabar, A.

Hamedoun, M., see Jabar, A.

Hamioud, F. \& Mubarak, A. A., The mechanical, optoelectronic and thermoelectric properties of $\operatorname{NiYSn}(Y=\mathrm{Zr}$ and $\mathrm{Hf})$ alloys

Han, D., Li, Y., He, Y., Qiu, S. \& Chen, H., Microstructure evolution and mechanical properties of 5083 aluminum alloy joints by ultrasonic soldering

Han, D.-Y., see Shi, P.-M.

Han, J. H., see Jiang, J.

Han, J., see Dai, B.

Han, L., see Li, J.

Han, X., see Guo, S.

Hang, Z., see Liu, Y.

Hang, Z., see Xi, N.

Hang, Z., see Zhang, M.

Hanh, P. T. M., see Ha, V. T.
B31 (2017) 1750264

B31 (2017) 1750203

B31 (2017) 1750203

B31 (2017) 1750019

B31 (2017) 1750235

B31 (2017) 1750127

B31 (2017) 1750141

B31 (2017) 1650250

B31 (2017) 1750135

B31 (2017) 1742002

B31 (2017) 1750208

B31 (2017) 1750035

B31 (2017) 1750170

B31 (2017) 1744040

B31 (2017) 1750113

B31 (2017) 1750186

B31 (2017) 1744075

B31 (2017) 1745006

B31 (2017) 1750169

B31 (2017) 1744038

B31 (2017) 1744024

B31 (2017) 1744047 
T.

Hantour, H. H., Investigation of optical properties and local structure of $\mathrm{Gd}^{3+}$ doped nanocrystalline $\mathrm{GeSe}_{2}$

Hao, R., see Zhou, W.

Hao, Y., see Hu, M.

Hao, Y., see Song, J.

Harroun, T. A., see Przedborski, $\mathrm{M}$

Hashmi, M. R.-U.-R., see Ahmed, $\mathrm{S}$.

Hassan, S. S. A., see AbdelRady, A. S.

Hassanabadi, H., see Onyeaju, M. C.

Hassani, H. R., see Fazeli, $\mathrm{M}$.

He, H., see Lee, W.

He, J., Tang, C., Liu, G. \& $\mathrm{Li}$, W., Effect of landslides on the structural characteristics of landcover based on complex networks

He, J., see Peng, H.

He, X., see Božović, I.

He, X., see Gasparov, V. A.

$\mathrm{He}, \mathrm{X}$., see Peng, $\mathrm{P}$.

He, Y., Wang, X., Hu, J., Zhou, Q. \& Chen, H., Effect of $\mathrm{Cu}$ content on exfoliation corrosion and electrochemical corrosion of A7N01 aluminum alloy in EXCO solution

He, Y., see Han, D.

He, Z., see Huang, A.

Heidarzadeh, H., see Jafari, $\mathrm{H}$.

Herbold, E. B., Stationary rarefaction waves in discrete materials with strain-softening behavior

Herojit Singh, L., see Panda, A.

Hieu, H. K., see Ha, V. T. T. Hikita, H., Ishikawa, H. \& Morigaki, K., A fractal process of hydrogen diffusion in a-Si:H with exponential energy distribution

Hlil, E. K., see Jabar, A.
B31 (2017) 1750141

B31 (2017) 1750075

B31 (2017) 1750242

B31 (2017) 1750192

B31 (2017) 1750222

B31 (2017) 1742011

B31 (2017) 1650243

B31 (2017) 1750091

B31 (2017) 1750119

B31 (2017) 1750059

B31 (2017) 1750039

B31 (2017) 1750156

B31 (2017) 1741012

B31 (2017) 1745005

B31 (2017) 1745016

B31 (2017) 1741006

B31 (2017) 1744005

B31 (2017) 1744040

B31 (2017) 1750087

B31 (2017) 1750002

B31 (2017) 1742005

B31 (2017) 1750033

B31 (2017) 1750141

B31 (2017) 1750060 B31 (2017) 1750035
Hofmeister, A. M., see Criss, E. M.

Hohenester, U., see Trügler,

A.

B31 (2017) 1750205

B31 (2017) 1740007

Honarasa, G., see Naseri, M.

Hong, J., Nonlinear entangled quantum dynamics in many-body systems with strong electron correlation

Hong, Q., see Shang, Y.-Q.

Hosseini, S., see Bordbar, G. $\mathrm{H}$.

Hou, D., see Yao, Y.

Hou, F., Hu, B., Tay, S. L., Wang, Y., Xiong, C. \& Gao, W., A new, bright and hard aluminum surface produced by anodization

Hou, F., see Bo, H.

Hou, F., see Tay, S. L.

Hou, F., see Wang, Y.

Hou, F., see Wang, Y.

Hou, H. \& Gao, W., Effects of $p b$ addition on microstructures and elemental distribution of AS-CAST AZ61 Mg alloy

Hou, H., see Gao, N.

Hou, H., see Zhang, C.

Hou, Q., Zhao, C. \& Qu, L., Effects of $V$ heavy doping on the magnetic and optical properties in anatase $\mathrm{TiO}_{2}$

Hou, Q., see Xu, Z.

Hou, Q.-Y., Li, W.-C., Qu, L.-F. \& Zhao, C.-W., Study on the effects of $G a$ $2 N$ high co-doping and preferred orientation on the stability, bandgap and absorption spectrum of $\mathrm{ZnO}$

Hou, X.-W., see Yuan, Q.

Hou, Y., see Li, J.

Houmad, M., Dakir, O., Benzidi, H., Mounkachi, O., El Kenz, A. \& Benyoussef, A., Magnetic behavior of Mn-doped silicon carbide nanosheet

Hsieh, W., see Tserkezis, C.
B31 (2017) 1742009

B31 (2017) 1750127

B31 (2017) 1741004

B31 (2017) 1750152

B31 (2017) 1750097

B31 (2017) 1750204

B31 (2017) 1744029

B31 (2017) 1744023

B31 (2017) 1744028

B31 (2017) 1744022

B31 (2017) 1744025

B31 (2017) 1744002

B31 (2017) 1750011

B31 (2017) 1750079

B31 (2017) 1650240

B31 (2017) 1750008

B31 (2017) 1750107

B31 (2017) 1750088

B31 (2017) 1741014

B31 (2017) 1750163

B31 (2017) 1740005 
Hu, B., see Hou, F.

$\mathrm{Hu}, \mathrm{B}$., see Wang, C.

$\mathrm{Hu}$, B., see Wang, Y.

$\mathrm{Hu}, \mathrm{B}$., see Wang, Y.

Hu, F., Zhu, Y., Shi, Y., Cai, J., Chen, L. \& Shen, S., An algorithm Walktrap-SPM for detecting overlapping community structure

Hu, G. C., see Cai, L. L.

$\mathrm{Hu}$, J., see He, Y.

$\mathrm{Hu}$, J., see Ji, Y.

Hu, M., Guo, K., Zhang, Z., Jiang, X., Li, K. \& Liu, D., The effect of positiondependent mass on nonlinear optical absorption coefficients and refractive index changes in a quantum well

Hu, M., Wei, H., Xiao, L., Zhang, K. \& Hao, Y., Temperature compensation effects of $\mathrm{TiO}_{2}$ on $\mathrm{Ca}\left[\left(\mathrm{Li}_{1 / 3} \mathrm{Nb}_{2 / 3}\right)_{0.8} \mathrm{Sn}_{0.2}\right] \mathrm{O}_{3-\delta}$ microwave dielectric ceramic

$\mathrm{Hu}, \mathrm{X}$, see Gao, W.

Hu, Y. F., see Sun, S. P.

Hu, Y., Chen, F., Chen, P. \& Tan, Y., The influence of passenger flow on the topology characteristics of urban rail transit networks

Hu, Y., see Fan, K.

Hua, J., see Yu, M.

Hua, J.-C., see Roy, S.

Hua, L., see Zhu, Q.-L.

Huang, A., Zang, G., He, Z. \& Guan, W., Comparative empirical analysis offlowweighted transit route networks in R-space and evolution modeling

Huang, B.-J. \& Chern, C.-H., Deconfinement of electric charges in hydrogen-bonded ferroelectrics

Huang, B.-J. \& Chern, C.-H., Nonrelativistic fermionic energy gap in the nonabelian gauge systems
B31 (2017) 1744029
B31 (2017) 1744055
B31 (2017) 1744022
B31 (2017) 1744025

B31 (2017) 1750121

B31 (2017) 1750072

B31 (2017) 1744005

B31 (2017) 1750188

B31 (2017) 1750009

B31 (2017) 1750192

B31 (2017) 1750042

B31 (2017) 1744081

B31 (2017) 1750181

B31 (2017) 1750080

B31 (2017) 1744027

B31 (2017) 1742007

B31 (2017) 1750136

B31 (2017) 1750087

B31 (2017) 1750130

B31 (2017) 1750126
Huang, C., see Zhong, M.M.

B31 (2017) 1750131

Huang, F. \& Liang, Y., Analysis and design of the system of a total digital si-gyroscope

Huang, G.-F., see Wu, J.

Huang, S. Y., see Sun, R. X.

Huang, W., see Chen, Z.

Huang, W., see Mamat, A.

Huang, W., see Zhu, X.

Huang, W.-Q., see Wu, J.

Huang, X., Cai, W.-M. \& Guo, H., Screening masses of photons interacting with a twospecies fermionic system in relativistic $B C S-B E C$ crossover

B31 (2017) 1750078

B31 (2017) 1744059

B31 (2017) 1744068

B31 (2017) 1744070

B31 (2017) 1750142

B31 (2017) 1744057

B31 (2017) 1744035

B31 (2017) 1744063

B31 (2017) 1750127

B31 (2017) 1750141

Hung, V. V., see Ha, V. T.

Huo, Q., Xu, Z. \& Qu, L., Effects on the magnetic and optical properties of Co-doped $\mathrm{ZnO}$ at different electronic states

Hussin, R., see Bulus, I.

Hussin, V., see Dey, S.

Iitaka, T., see Li, Z.

Ikot, A. N., see Onyeaju, M. C.

Iliass, T., see Cambui, D. S. Indu, B. D., see Sharma, R.

Iqbal, R., Ali, Z., JalaliAsadabadi, S. \& Ahmad, I., Electron correlation and spin-orbit coupling effects in scandium intermetallic compounds ScTM $(T M=C o, R h, I r$, $\mathrm{Ni}, \mathrm{Pd}, \mathrm{Pt}, \mathrm{Cu}, \mathrm{Ag}$ and Au)

Iqbal, Z., see Zulfiqar

Ishikawa, H., see Hikita, H.

Ishikawa, S., see Furuta, T.

Itoh, S., see Furuta, T.

Iyakutti, K., Rajeswarapalanichamy, R., Surya,
B31 (2017) 1750247

B31 (2017) 1750101

B31 (2017) 1650248

B31 (2017) 1750217

B31 (2017) 1750119

B31 (2017) 1750073

B31 (2017) 1750225

B31 (2017) 1750263

B31 (2017) 1750234

B31 (2017) 1750060

B31 (2017) 1742006

B31 (2017) 1742006 
V. J. \& Kawazoe, Y., Quantum scar and breakdown of universality in graphene: A theoretical insight

Jabar, A., Masrour, R., Benyoussef, A. \& Hamedoun, M., Magnetic properties and binder $\mathrm{cu}$ mulants of a mixed spin-2 and spin-5/2 ising diamond chain

Jabar, A., Masrour, R., Hamedoun, M., Benyoussef, A. \& Hlil, E. K., Magnetic properties of zigzag metallic chains: A Monte Carlo study

Jafar Hussain, M., see Abbas, G.

Jafari, H., Heidarzadeh, H., Rostami, A., Rostami, G. \& Dolatyari, M., Significant performance enhancement in continuous wave terahertz photomixers based on fractal structures

Jalali-Asadabadi, S., see Iqbal, R.

Jalali-Asadabadi, S., see Khan, I.

Jalilian, J., see Naseri, M.

Jani, M. \& Ray, A., Firstprinciples study of electronic and optical properties of solid-solution $\mathrm{ZnO}_{1-x} \mathrm{~S}_{x}$

Javaheri, S., Boochani, A., Babaeipour, M. \& Naderi, S., Electronic and optical properties of ALN under pressure: DFT calculations

Javaheri, S., Boochani, A., Babaeipour, M. \& Naderi, S., Electronic and optical properties of $\mathrm{GaN}$ under pressure: DFT calculations

Jayannavar, A. M., see Bandyopadhyay, M.

Jayaprakash, K. R., see Starosvetsky, Y.

Jean, M. S., see Coste, C.
B31 (2017) 1750257

B31 (2017) 1750208

B31 (2017) 1750035

B31 (2017) 1750193

B31 (2017) 1750002

B31 (2017) 1750263

B31 (2017) 1750148

B31 (2017) 1750044

B31 (2017) 1750175

B31 (2017) 1650255

B31 (2017) 1750261

B31 (2017) 1750189

B31 (2017) 1742001

B31 (2017) 1742003
Ji, C.-Y., see Shen, F.-F.

$\mathrm{Ji}$, J., see Yang, $\mathrm{T}$.

Ji, L.-B. \& Chen, F., Process optimization of rolling for zincked sheet technology using response surface methodology and genetic algorithm

Ji, W., see Peng, H.

Ji, X., see Liu, S.

Ji, Y. \& Hu, J., Application of the recursion-transform method in calculating the resistance of an $M \times N$ cobweb resistor network with an arbitrary boundary

Ji, Y., Xie, Y., Zhu, S. \& Yan, B., Effects of $W$ on microstructure and hightemperature oxidation behavior of ferritic stainless steel weldment

Jia, B., see Ye, Y.

Jia, L., see Su, F.

Jia, P., Tan, H., Liu, K. \& Gao, W., Adsorption behavior of methylene blue by bone char

Jia, Y., Li, S., Qi, W., Wang, M., Li, Z. \& Wang, Z., Thermal stability of marks gold nanoparticles: A molecular dynamics simulation

Jia, Y.-T., see Zhang, J.

Jian, Z., see Wang, L.

Jiang, D., Chen, Y., Gu, X., Xie, L. \& Chen, L., Efficient and universal quantum key distribution based on chaos and middleware

Jiang, G., see Shi, S.-P.

Jiang, J., Han, J. H., Zhang, R. \& Li, W., The transition point of the Chinese multilayer air transportation networks

B31 (2017) 1650259

B31 (2017) 1750028

B31 (2017) 1744017

B31 (2017) 1741012

B31 (2017) 1650254

B31 (2017) 1750188

B31 (2017) 1744042

B31 (2017) 1744100

B31 (2017) 1750027

B31 (2017) 1744099

B31 (2017) 1741001

B31 (2017) 1745020

B31 (2017) 1750254

B31 (2017) 1650264

B31 (2017) 1750022

Jiang, Q. W., see Li, M.

Jiang, X., see Dai, G.

Jiang, X., see Hu, M.

Jiang, Y. H., see Qin, X. M.

Jiang, Y., see Qi, Y.

Jiang, Y., see Sun, S. P.

Jiang, Y., see Yang, K.
B31 (2017) 1750186

B31 (2017) 1744014

B31 (2017) 1744087

B31 (2017) 1750009

B31 (2017) 1744012

B31 (2017) 1745022

B31 (2017) 1744081

B31 (2017) 1744030 
Jiang, Y., see Yao, C.

Jiang, Y., see Yao, C.

Jiang, Y., see Ye, Y.

Jiang, Z. \& Tie, S., Preparation and thermal properties of Glauber's salt-based phase-change materials for QinghaiTibet plateau solar greenhouses

Jiang, Z.-Q., see $\mathrm{Xu}, \mathrm{H} .-\mathrm{C}$. Jiao, M., Yang, K., Ren, D., Diao, Q., Cao, J., Liu, H. \& Yu, M., The solution curing performance of high-ortho epoxy phenolic fibers

Jiao, M., see Yang, K.

Jiao, P., Lyu, H., Li, X., Yu, W. \& Wang, W., Temporal community detection based on symmetric nonnegative matrix factorization

Jin, C.-Q., see Zhang, J.

Jin, L. W., see Lei, W. Y.

Jin, Q., see Dong, H. M.

Jin, X., see Peng, J. F.

Jing, C., see Ma, W.

Jones, M. I., see Yin, L.

Jong, C.-S., see Chakraborty, A.

José, J. V., Duality, gauge symmetries, renormalization groups and the BKT transition

Joudeh, B. R., see AlMaaitah, A. F.

Kabanov, V. V., Magnetic quantum oscillations in doped antiferromagnets

Kandemir, B. S. \& Gökçek, N., Velocity renormalization in graphene: The role of trigonal warping and electron-phonon coupling effects

Kaneyoshi, T., Amorphization in an ising nanowire

Karimian, N., see Golivand, M. B.

Kashyap, R., Westley, A., Datta, A. \& Sen, S., Early time evolution of a localized nonlinear excitation
B31 (2017) 1744004
B31 (2017) 1744082
B31 (2017) 1744100

B31 (2017) 1744085

B31 (2017) 1750048

B31 (2017) 1744090

B31 (2017) 1744102

B31 (2017) 1750102

B31 (2017) 1745020

B31 (2017) 1745021

B31 (2017) 1750098

B31 (2017) 1744020

B31 (2017) 1730002

B31 (2017) 1744080

B31 (2017) 1650241

B31 (2017) 1730001

B31 (2017) 1750202

B31 (2017) 1745015

B31 (2017) 1750235

B31 (2017) 1750071

B31 (2017) 1750003 in the $\beta$-FPUT chain

Katou, K., see Furuta, T.

Katriel, J., Comment on the spherical quantum dot with interaction effects

Katsuragi, H., see Furuta, T.

Kawazoe, Y., see Iyakutti, $\mathrm{K}$.

Kazzaz, H. A., see Yarmohammadi, M.

Ke, P. L., see Zuo, X.

Ke, S. H., see Zhang, J.

Kenfack-Jiotsa, A., see Djoufack, Z. I.

Kenkre, V. M. \& Chase, M., Approach to equilibrium of a quantum system and generalization of the Montroll-Shuler equation for vibrational relaxation of a molecular oscillator

Keshavarz, A., see Naseri, M.

Kevrekidis, P. G., Carretero-González, R. \& Frantzeskakis, D. J., Stability of single and multiple matter-wave dark solitons in collisionally inhomogeneous BoseEinstein condensates

Kh. Ramadan, see Alajerami, Y. S. M.

Khalifa, Z., see Aly, A. H.

Khalil, E. M., see Abdalla, M. S.

Khan, A. \& Tanatar, B., Effect of weak disorder on the BCS-BEC crossover in a two-dimensional fermi gas

Khan, I., Shehzad, N., Ahmad, I., Ali, Z. \& Jalali-Asadabadi, S., First-principle studies of the optoelectronic properties of $\mathrm{ASnF}_{3}(\mathrm{~A}=\mathrm{Na}, \mathrm{K}$, $\mathrm{Rb}$ and $\mathrm{Cs}$ )

Khan, S. H., see Faizan, M.

Khayatian, S. A., see Gh. Khorrami, H.

Kheirandish, F., see Nasr, Z.

Khordad, R., Optical properties of wedge-shaped quantum dots under
B31 (2017) 1742014

B31 (2017) 1742006

B31 (2017) 1750115

B31 (2017) 1742006

B31 (2017) 1750257

B31 (2017) 1750053

B31 (2017) 1744032

B31 (2017) 1750032

B31 (2017) 1750140

B31 (2017) 1750244

B31 (2017) 1750152

B31 (2017) 1742013

B31 (2017) 1750171

B31 (2017) 1750147

B31 (2017) 1750211

B31 (2017) 1750066

B31 (2017) 1750148

B31 (2017) 1750253

B31 (2017) 1750194

B31 (2017) 1750122 
rashba spin-orbit interaction

Khorrami, M., see Tajik, F.

Khorsand Zak, A., see Gh. Khorrami, $\mathrm{H}$.

Khosravi, H., Boochani, A., Rasolian, G., Solaymani, S. \& Naderi, S., Dft study of elastic, half-metallic and optical properties of $\mathrm{Co}_{2} \mathrm{~V}(\mathrm{Al}, \mathrm{Ge}, \mathrm{Ga}$ and $\mathrm{Si})$ compounds

Khurgin, J. B., see Tserkezis, C.

Khusyainov, D. I., Dekeyser, C., Buryakov, A. M., Mishina, E. D., Galiev, G. B., Klimov, E. A., Pushkarev, S. S. \& Klochkov, A. N., Ultrafast carrier dynamics in LT-GaAs doped with si delta layers

Klimov, E. A., see Khusyainov, D. I.

Klochkov, A. N., see Khusyainov, D. I.

Kodeh, F. S., see Alajerami, Y. S. M.

Kofane, T. C., see Ngounga Makoundit, G. J.

Köksal, K., see Öncan, M.

Kolesnikov, D. V., Sadykova, O. G. \& Osipov, V. A., Enhancement of thermoelectric figure of merit in zigzag graphene nanoribbons with periodic edge vacancies

Komorowski, P. G. \& Cottam, M. G., Electronic modes in carbon nanotubes with single and double impurity sites

Kompany, A., see Gh. Khorrami, $\mathrm{H}$.

Kong, Y., see Diao, Y.

Kong, Y., see Xia, S.

Kou, S.-P., Kelvin wave and knot dynamics on entangled vortices

Kouhi, M., Nonlinear optical absorption in the core shell nanowire

Koç, F., see Öncan, M.
B31 (2017) 1750055 B31 (2017) 1750258

B31 (2017) 1750194

B31 (2017) 1750109

B31 (2017) 1740005

B31 (2017) 1750195

B31 (2017) 1750195

B31 (2017) 1750195

B31 (2017) 1750171

B31 (2017) 1750150

B31 (2017) 1750095

B31 (2017) 1750124

B31 (2017) 1750220

B31 (2017) 1750194

B31 (2017) 1750084

B31 (2017) 1650252

B31 (2017) 1750241

B31 (2017) 1750164 B31 (2017) 1750095
Krawiecki, A., Stochastic resonance in the majority vote model on regular and small-world lattices

B31 (2017) 1750214

B31 (2017) 1742008

Kress, T., see Christov, I. C.

Krstovska, D., Ultrasonic wave generation in twoband organic conductors due to thermoelectric effect

Kuang, Z., see Yan, J. G.

Kui, M.-Q., see Tan, X.-H.

Kumar, A., see Diwaker

Kumar, R., see Dhiman, S.

Kushwaha, A. K., Vibrational, mechanical and thermodynamical properties of $\mathrm{RE}_{2} \mathrm{Ti}_{2} \mathrm{O}_{7}$ $(R E=S m, G d, D y, H o, E r$ and $\mathrm{Yb}$ ) pyrochlores

$\mathrm{L}$, see Fan, K.

Lai, M., see $\mathrm{Xu}, \mathrm{C}$.

Lakshmanan, A., see Alexandar, A.

Lamzabi, S., see Bamaarouf, O.

Lamzabi, S., see Lazfi, S.

Lazfi, S., Lamzabi, S., Rachadi, A. \& EzZahraouy, H., The impact of neighboring infection on the computer virus spread in packets on scale-free networks

Lee, G. H., see Tegafaw, T.

Lee, M. H., Chemical potential, Gibbs-Duhem equation and quantum gases

Lee, P., In memory of kerson huang: Teacher, colleague and friend

Lee, S. H., see Tegafaw, T.

Lee, T., Resonant multilead point-contact tunneling: boundary state formulation

Lee, W., He, H. \& Chen, M., Theoretical investigations of the $\mathrm{Au}^{+}+\mathrm{H}_{2}$ reactive scattering by the time-dependent quantum wave packet method

Lei, J., Zhi, M. H., Li, X. W., Liang, T. \& Qiao, D. H., Design of microwave
B31 (2017) 1750250

B31 (2017) 1650263

B31 (2017) 1750219

B31 (2017) 1650249

B31 (2017) 1750092

B31 (2017) 1750145

B31 (2017) 1741007

B31 (2017) 1744059

B31 (2017) 1750174

B31 (2017) 1750182

B31 (2017) 1750228

B31 (2017) 1750228

B31 (2017) 1750014

B31 (2017) 1750104

B31 (2017) 1777001

B31 (2017) 1750014

B31 (2017) 1750024

B31 (2017) 1750039 
signal source for cs chipscale atomic clock

Lei, J., see Song, Y.

Lei, R., see Ding, M.

Lei, W. Y., Qian, N., Zheng, J., Jin, L. W., Zhang, Y. \& Deng, Z. G., Dynamic motion modes of high temperature superconducting maglev on a 45-m long ring test line

Lerch, S. \& Reinhard, B. M., Spectral signatures of charge transfer in assemblies of molecularly-linked plasmonic nanoparticles

León, M., see Durante Rincón, C. A.

Li, A., see Zhang, F.

Li, C., see Chen, W.

Li, D. H., see Yan, J. G.

Li, D., see Guo, Q.

Li, D., see Zhao, X.

Li, F., see Chen, Z.

Li, F., see Fu, D.

Li, F., see Zhou, W.

Li, H. T., see Sun, R. X.

Li, H. \& Zou, J., Study of 2219 aluminum alloy using direct current A-TIG welding

Li, H., Wang, J., Yi, G., Deng, B. \& Zhou, H., Eeg-based functional networks evoked by acupuncture at ST 36: A data-driven thresholding study

Li, H., see $\mathrm{Su}, \mathrm{F}$.

Li, H.-J., Cheng, Q., Mao, H.-J., Wang, H. \& Chen, J., Uncovering the effective interval of resolution parameter across multiple community optimization measures

Li, J., Li, X., Tang, J., Gong, T. \& Han, L., Numerical study on ac loss in superconducting bulk caused by various magnetic wave conditions

Li, J., Zhao, D., Hou, Y. \& Sun, B., Power coupling
B31 (2017) 1741010

B31 (2017) 1750243

B31 (2017) 1744096

B31 (2017) 1745021

B31 (2017) 1740002

B31 (2017) 1750246

B31 (2017) 1750223

B31 (2017) 1744031

B31 (2017) 1650263

B31 (2017) 1745001

B31 (2017) 1750183

B31 (2017) 1744069

B31 (2017) 1750172

B31 (2017) 1750242

B31 (2017) 1745014

B31 (2017) 1744043

B31 (2017) 1750187

B31 (2017) 1750238

B31 (2017) 1750041

B31 (2017) 1745006 characteristics between fbg and back-scattering signals

Li, J., see Guo, S.

Li, J., see Yang, K.

Li, K., see Hu, M.

Li, K., see Shao, F.

Li, M. \& Jiang, Q. W., The effect of strain rates on tensile deformation of ultrafine-grained copper

Li, M., see Wan, Y.

Li, Q., see Peng, T.

Li, Q., see Qin, L.

Li, Q., see Shi, P.-M.

Li, Q., see Wang, C.

Li, Q., see Yao, C.

Li, Q.-Q., see Zheng, B.-X.

Li, R., see Xu, G.

Li, R., see Xu, G.

Li, S., see Jia, Y.

Li, S., see Peng, H.

Li, S.-B., see Liu, Y.

Li, S.-L., Li, Y.-L., Li, Y.-J., Gong, P. \& Fang, X.-Y., Different roles of carbon and silicon vacancies in silicon carbide bulks and nanowires

Li, W., Sun, Y., Liu, Y. \& Zhao, D., Analyzing synchronization of timedelayed complex dynamical networks with periodic on-off coupling

Li, W., see Feng, L.

Li, W., see He, J.

Li, W., see Jiang, J.

Li, W.-C., see Hou, Q.-Y.

Li, W.-M., see Zhang, J.

Li, X. P., see Sun, S. P.

Li, X. W., see Lei, J.

Li, X., Zhao, X., Lv, F., Liu, F. \& Wang, Y., Improved corrosion resistance of new Fe-based amorphous alloys

Li, X., see Cao, X.

Li, X., see Cheng, X.

Li, X., see Cheng, X.

Li, X., see Gao, W.

Li, X., see Jiao, P.

Li, X., see Liu, Y.

Li, X., see Li, J.

Li, X., see Wang, B.
B31 (2017) 1741014

B31 (2017) 1750169

B31 (2017) 1744030

B31 (2017) 1750009

B31 (2017) 1750010

B31 (2017) 1744014

B31 (2017) 1744070

B31 (2017) 1750016

B31 (2017) 1744073

B31 (2017) 1750113

B31 (2017) 1744055

B31 (2017) 1744082

B31 (2017) 1775001

B31 (2017) 1750030

B31 (2017) 1750034

B31 (2017) 1741001

B31 (2017) 1741012

B31 (2017) 1744091

B31 (2017) 1750173

B31 (2017) 1750210

B31 (2017) 1750185

B31 (2017) 1750156

B31 (2017) 1750186

B31 (2017) 1750107

B31 (2017) 1745020

B31 (2017) 1744081

B31 (2017) 1741010

B31 (2017) 1744010

B31 (2017) 1744071

B31 (2017) 1744060

B31 (2017) 1744061

B31 (2017) 1750042

B31 (2017) 1750102

B31 (2017) 1744041

B31 (2017) 1745006

B31 (2017) 1750139 
Li, X., see Zhang, L.

Li, X.-P., see Tan, X.-H.

Li, X.-X. \& Chen, G., Effect of electron-electron interaction on polarization and dissociation of the charged oligomer after single-photon absorption

Li, Y. F., Tang, S. L., Gao, Y. M., Ma, S. Q., Zheng, Q. L. \& Cheng, Y. H., Mechanical and thermodynamic properties of intermetallic compounds in the Ni-Ti system

Li, Y. H., see Liu, C. G.

Li, Y. \& Yang, S., Doping effect on exciton binding energy in semiconductor quantum well

Li, Y., see Han, D.

Li, Y., see Pu, J.

Li, Y., see Sun, L.

Li, Y., see Yao, C.

Li, Y., see Zhu, Z.

Li, Y., Quantum AdaBoost algorithm via cluster state

Li, Y.-J., see Li, S.-L.

Li, Y.-L., see Li, S.-L.

Li, Z., Xu, D.-D., Ning, S.-Y., Su, H., Iitaka, T., Tohyama, T. \& Zhang, J.-X., Predicted weyl fermions in magnetic GdBi and GdSb

Li, Z., see Chen, Y.

Li, Z., see Jia, Y.

Li, Z.-Q., see Zheng, B.-X.

Liang, T., see Lei, J.

Liang, Y., see Huang, F.

Liang, Y., see Peng, T.

Liao, H., Yang, G., Zeng, M. \& Wang, X., The solidification and precipitation behaviors of AE42 magnesium alloy

Liao, L., Peng, X., Shi, J. \& Guo, Y., Graph statebased quantum authentication scheme

Liao, W., see Ye, Y.

Lin, G., see Yuan, X.

Lin, L., Wang, H. \& Zhong,
B31 (2017) 1744007

B31 (2017) 1750219

B31 (2017) 1750256

B31 (2017) 1750161 B31 (2017) 1750184

B31 (2017) 1750004

B31 (2017) 1744040

B31 (2017) 1744046

B31 (2017) 1750012

B31 (2017) 1744082

B31 (2017) 1744035

B31 (2017) 1750040

B31 (2017) 1750173

B31 (2017) 1750173

B31 (2017) 1750217

B31 (2017) 1744057

B31 (2017) 1741001

B31 (2017) 1775001

B31 (2017) 1741010

B31 (2017) 1741008

B31 (2017) 1750016

B31 (2017) 1744006

B31 (2017) 1750067

B31 (2017) 1744100

B31 (2017) 1650244
S., Stochastic resonance for a fractional oscillator with random trichotomous mass and random trichotomous frequency

Lin, Y., see Pan, Y.

Lindenberg, K., see Rosas, A.

Lionel, T. K., Tchoffo, M., Fouokeng, G. C. \& Fai, L. C., Effects of static noise on the dynamics of quantum correlations for a system of three qubits

Liu, C. G., Zhang, J., Chen, L. J., Wen, J., Dong, L. Y., Yang, D. Y. \& Li, Y. H., The structural parameters, structural stability and bulk modulus in $\mathrm{RE}_{2} \mathrm{Sn}_{2} \mathrm{O}_{7}$ by firstprinciples calculations

Liu, C., Yamaguchi, Y. \& Dou, X., Molecular sieving polymer for DNA/RNA separation in capillary electrophoresis

Liu, C., see Liu, S.

Liu, C., see Su, F.

Liu, C., see Wang, Q.

Liu, C.-Z., see An, N.

Liu, D., see $\mathrm{Hu}, \mathrm{M}$.

Liu, D., see Yang, T.

Liu, F., see Li, X.

Liu, G., see He, J.

Liu, H., see Feng, L.

Liu, H., see Feng, L.

Liu, H., see Guo, Q.

Liu, H., see Jiao, M.

Liu, H.-C., see Yan, X.-A.

Liu, J., see Zhang, W.

Liu, J.-F., see Wang, Y.-C.

Liu, K., see Dai, B.

Liu, K., see Jia, P.

Liu, L. \& Luo, G., Routing optimization in networks based on traffic gravitational field model

Liu, L., see Diao, Y.

Liu, L., see Xia, S.

Liu, P., Fan, G., Liu, Y., Yang, L. \& Miao, X., Effect of aluminum trace dimension on electromigration failure in flip-
B31 (2017) 1750231

B31 (2017) 1750096

B31 (2017) 1742016

B31 (2017) 1750046

B31 (2017) 1750184

B31 (2017) 1744094

B31 (2017) 1650254

B31 (2017) 1750238

B31 (2017) 1750057

B31 (2017) 1750089

B31 (2017) 1750009

B31 (2017) 1750028

B31 (2017) 1744010

B31 (2017) 1750156

B31 (2017) 1650239

B31 (2017) 1750185

B31 (2017) 1745001

B31 (2017) 1744090

B31 (2017) 1650245

B31 (2017) 1750005

B31 (2017) 1744067

B31 (2017) 1744075

B31 (2017) 1744099

B31 (2017) 1750074

B31 (2017) 1750084

B31 (2017) 1650252 
chip package

Liu, Q.-Q., see Zhang, J.

Liu, R., see Yang, K.

Liu, S., Ji, X., Liu, C. \& Bai, Y., Similarity indices based on link weight assignment for link prediction of unweighted complex networks

Liu, S., see Peng, T.

Liu, X. \& Tie, S., Weather resistance of $\mathrm{CaSO}_{4}$. $1 / 2 \mathrm{H}_{2} \mathrm{O}$-based sand-fixation material

Liu, X., see Mamat, A.

Liu, X., see Qi, Y.

Liu, Y. \& Gao, W., Decoration of $\mathrm{ZnO}$ nanorod arrays by $\mathrm{Cu}$ nanocrystals via magnetron sputtering

Liu, Y., Chen, H., Liu, Y. \& Hang, Z., Simulated haz continuous cooling transformation diagram of a bogie steel of high-speed railway

Liu, Y., Gou, G., Chen, J., Chen, H., Wang, W., Li, $\mathrm{X}$., Che, X. \& Wang, Y., Effect of composition on the tensile properties and fracture toughness of A7N01S-T5 aluminum alloys welded joints

Liu, Y., Li, S.-B., Yao, P. \& Zhang, Q.-M., Synthesis of organic soluble poly(substitutedaniline) from 2-methyl6-ethylaniline tar

Liu, Y., see Liu, P.

Liu, Y., see Liu, Y.

Liu, Y., see Li, W.

Liu, Y., see Sun, L.

Liu, Y., see Xi, N.

Liu, Y., see Zhang, J.

Liu, Y.-L., see Shi, S.-P.

Liu, Y.-W., see Yan, X.-A.

Liu, Z., see Fan, K.

Liu, Z., see Fan, K.

Lomsadze, R. A., Gochitashvili, M. R. \& Ya. Kezerashvili, R., Precision measurements of cross-sections for inelas-

\author{
B31 (2017) 1741002 \\ B31 (2017) 1745020 \\ B31 (2017) 1744102 \\ B31 (2017) 1650254 \\ B31 (2017) 1750016 \\ B31 (2017) 1744084 \\ B31 (2017) 1745002 \\ B31 (2017) 1745022
}

B31 (2017) 1744050

B31 (2017) 1744038

\section{B31 (2017) 1744041}

B31 (2017) 1744091

B31 (2017) 1741002

B31 (2017) 1744038

B31 (2017) 1750210

B31 (2017) 1750012

B31 (2017) 1744024

B31 (2017) 1745020

B31 (2017) 1750022

B31 (2017) 1650245

B31 (2017) 1741007

B31 (2017) 1741011 tic processes in collisions of alkali metal ions with atoms of rare gases

Long, X., see Song, J.

Longhi, S., Non-hermitian interaction of a discrete state with a continuum

Lorestaniwiess, Z., see Rashidian, Z.

Lu, D.-F., see Shen, F.-F.

Lu, G., see Zhang, X.

Lu, H. \& Yan, G.-A., Fidelity analysis of cyclic threelevel system-based quantum routing

Lu, J., see Zulfiqar

Lu, S., see Yao, C.

Lu, X., see Zhang, W.

Lü, L., see $\mathrm{Xu}, \mathrm{G}$.

Luan, X., see Ye, Y.

Luitel, H. \& Sanyal, D., $a b$ initio calculation of magnetic properties in $B, A l$, C, Si, N, P and As-doped rutile $\mathrm{TiO}_{2}$

Luo, D. \& Yin, L., Critical temperature of pair condensation in a dilute bose gas with spin-orbit coupling

Luo, G., see Liu, L.

Luo, H., see Zhang, W.

Luo, P., see Guan, X.

Lv, B. \& Guo, W., The spin pairing symmetry of $d$-wave superconductor indicated by tunneling spectroscopy

Lv, F., see Li, X.

Lv, J., see Cai, J.

Lv, J., see Wang, X.

Lyu, H., see Jiao, P.

Ma, F., see Gao, Y.

Ma, J., Fan, X., Ni, J., Zhu, X. \& Xiong, C., Multiscale retinex with color restoration image enhancement based on gaussian filtering and guided filtering

Ma, J., Wu, F. \& Wang, C., Synchronization behaviors of coupled neurons under electromagnetic radiation
B31 (2017) 1650257

B31 (2017) 1750222

B31 (2017) 1750249

B31 (2017) 1750043

B31 (2017) 1650259

B31 (2017) 1740003

B31 (2017) 1750190

B31 (2017) 1750234

B31 (2017) 1744082

B31 (2017) 1750005

B31 (2017) 1750030

B31 (2017) 1744100

B31 (2017) 1750227

B31 (2017) 1745012

B31 (2017) 1750074

B31 (2017) 1750005

B31 (2017) 1744019

B31 (2017) 1745017

B31 (2017) 1744010

B31 (2017) 1744078

B31 (2017) 1744021

B31 (2017) 1750102

B31 (2017) 1750021

B31 (2017) 1744077

B31 (2017) 1650251 
Ma, J., see Wang, B.

Ma, J., see Xiao, J.

Ma, J., see Xiong, C.

Ma, J., see Zhang, G.

Ma, J.-X., see Chen, L.

Ma, S. Q., see Li, Y. F.

Ma, W. \& Jing, C., Firstprinciples study on hydrogen storage in $\mathrm{Al}-$, Ca-, Mn-doped MgNi clusters

Ma, X. S., Cheng, M.-T. \& Ying, Q., Dynamics of measurement-induced nonlocality of quantum states in a structured environment

Ma, Y., see Xu, G.

Ma, Y.-L., see Shang, Y.-Q.

Maabed, S., see Benmakhlouf, A.

Mahmood, A., see Faizan, M.

Maillot, M., see Ariyanti, D.

Maisnam, M., see Nilima, $\mathrm{N}$.

Maity, A. \& Sen, P., Density functional study of metalphosphorene interfaces

Malyarenko, A. \& OstojaStarzewski, M., Fractal planetary rings: Energy inequalities and random field model

Mamani, E., CalcinaNogales, M. \& Sanjinés, D., Long-range effective interactions in a lattice in the semiclassical approximation

Mamat, A., Qi, Y., Rozi, A., Liu, X., Huang, W., Wang, T., Ni, H. \& Gao, J., Oxygen defect influenced gas sensing properties of $\mathrm{ZnO}$ polyhedral structures

Mandal, A., see Pan, S.

Mansour, M. \& Daoud, M., Stabilizer codes and equientangled bases from phase states

Mao, H.-J., see Li, H.-J.

Mao, Z.-L., see Tan, X.-H.

Marchewka, M., Finitedifference method applied for eight-band $k p$ model
B31 (2017) 1750139
B31 (2017) 1744062
B31 (2017) 1744076
B31 (2017) 1750180
B31 (2017) 1744063
B31 (2017) 1750161

B31 (2017) 1730002

B31 (2017) 1650260

B31 (2017) 1750034

B31 (2017) 1741004

B31 (2017) 1750086

B31 (2017) 1750253

B31 (2017) 1744095

B31 (2017) 1750083

B31 (2017) 1750077

B31 (2017) 1750236

B31 (2017) 1750116

B31 (2017) 1745002

B31 (2017) 1750019

B31 (2017) 1750132

B31 (2017) 1750041

B31 (2017) 1750219 for $\quad \mathrm{Hg}_{1-x} \mathrm{Cd} d_{x} \mathrm{Te} / \mathrm{HgTe}$ quantum well

B31 (2017) 1750137

Marcillo, F., Villamagua, L. \& Stashans, A., Analysis of electrical and magnetic properties of zinc oxide: A quantum mechanical study

Mardaani, M., see Shariati, A.

Mariscal-Sanchez, A. \& Sandoval-Villalbazo, A., Evolution of statistical averages: An interdisciplinary proposal using the Chapman-Enskog method

Marsiglio, F., see Souza, T. X. R.

Martikainen, J.-P., see Nečada, M.

Masoudi, A. A., see Tajik, F.

Masrour, R., see Jabar, A.

Masrour, R., see Jabar, A.

Mauro, M. D., Esposito, S. \& Naddeo, A., Phase time and transmission probability in the traversal of a PT-symmetric potential: The case of an electromagnetic waveguide

Md. Sohel, A., see Pan, S.

Medina, J. E., see Durante Rincón, C. A.

Meng, M., Wang, Z. B., Wang, X. \& Chen, Y., Creep-caused fracture of pbsn solder joint

Meng, Q., see Bai, Y.

Meng, X., see $\mathrm{Su}, \mathrm{C}$.

Meng, Y., see Deng, W.

Mhareb, M. H. A., see Alajerami, Y. S. M.

Miao, J., see Xiao, F.

Miao, X., see Liu, P.

Mignani, R., see Cardone, F.

Mirabbaszadeh, K., see Yarmohammadi, M.

Mishina, E. D., see Khusyainov, D. I.

Mishra, A. C. \& Giri, R., Micromagnetic simulation study of magnetization reversal in torus-shaped permalloy nanorings
B31 (2017) 1750111

B31 (2017) 1750199

B31 (2017) 1750143

B31 (2017) 1745003

B31 (2017) 1740006

B31 (2017) 1750258

B31 (2017) 1750208

B31 (2017) 1750035

B31 (2017) 1750213

B31 (2017) 1750019

B31 (2017) 1750246

B31 (2017) 1744008

B31 (2017) 1750036

B31 (2017) 1744045

B31 (2017) 1744068

B31 (2017) 1750171

B31 (2017) 1744026

B31 (2017) 1741002

B31 (2017) 1750168

B31 (2017) 1750045

B31 (2017) 1750195

B31 (2017) 1750162 
Mohamed, S. H., see Aly, A. $\mathrm{H}$.

Mohammadpour, H., see Esmailpour, $\mathrm{M}$.

Mohammadpour, H., see Eyni, Z.

Mohan, T. R. K., see Tiwari, M.

Mok, J. W., see Westley, A.

Monti, M., see Cardone, F.

Mookerjee, A., see Sadhukhan, B.

Moradian, R., see Chegel, $\mathrm{R}$.

Morais, C. W., De Freitas, D. N., Mota, A. L. \& Bastone, E. C., Phase transition and monopole densities in a nearest neighbor two-dimensional spin ice model

Morigaki, K., see Hikita, H.

Mosaad, M. M., see Ghali, M.

Mota, A. L., see Morais, C. W.

Moubissi, A. B., see Ngounga Makoundit, G. J.

Mounkachi, O., see Houmad, M.

Mousavi, M., see Gh. Khorrami, $\mathrm{H}$.

Movahhedian, H., see Zad, H. A.

Mubarak, A. A., see Hamioud, F.

Muley, A., see Ghosh, S.

Murthy, K. S. R., see Chaitanya Varma, $M$.

Musari, A. A., see Olajire, B. A.

Muthuganesan, R. \& Sankaranarayanan, R., Nonlocal correlation in heisenberg spin models

Naddeo, A., see Mauro, M. D.

Naderi, A., see Tahne, B. A.

Naderi, A., Improvement in the performance of graphene nanoribbon $p$ $i$-n tunneling field effect transistors by applying lightly doped profile on drain region

B31 (2017) 1750239

B31 (2017) 1650250

B31 (2017) 1750230

B31 (2017) 1742012

B31 (2017) 1742002

B31 (2017) 1750168

B31 (2017) 1750218

B31 (2017) 1750158

B31 (2017) 1750237

B31 (2017) 1750060

B31 (2017) 1750037

B31 (2017) 1750237

B31 (2017) 1750150

B31 (2017) 1750163

B31 (2017) 1750194

B31 (2017) 1750094

B31 (2017) 1750170

B31 (2017) 1750207

B31 (2017) 1750063

B31 (2017) 1750134

B31 (2017) 1750166

B31 (2017) 1750213

B31 (2017) 1650242
Naderi, S., see Javaheri, S.

Naderi, S., see Javaheri, S.

Naderi, S., see Khosravi, H.

Nagata, K., see Batle, J.

Nagatsu, M., see Shao, Z.

Nagaty, A., see Aly, A. H.

Najafi, H., see Bahari, A.

Nakamura, T., see Batle, J.

Namiot, V. A. \& Yu. Shchurova, L., On a barrier anti-zeno effect in a special model allowing for an analytical solution

Narikiyo, O., Spectral function method for hall conductivity of incoherent metals

Naseem, S., see Rauf, A.

Nasehnia, F. \& Seifi, M., Elastic and electronic properties of oxygen plasma-treated graphene sheets from first principles

Naseri, M., Jalilian, J. \& Reshak, A. H., Electronic and optical properties of pentagonal- $b_{2} C$ monolayer: A first-principles calculation

Naseri, M., Keshavarz, A. \& Honarasa, G., Imaging properties of a nonlinear multilayered superlens

Nasr, Z. \& Kheirandish, F., On the localized quantum oscillators in a common heat bath

Nayak, A., see Sadhukhan, B.

Nayak, P., see Sahoo, J.

Nečada, M., Martikainen, J.P. \& Törmä, P., Quantum emitter dipole-dipole interactions in nanoplasmonic systems

Ngounga Makoundit, G. J., Ekogo, T. B., Moubissi, A. B., Ben-Bolie, G. H. \& Kofane, T. C., Effects of three-body interactions on the instabilities and self-oscillations of the four-wave mix-
B31 (2017) 1750248

B31 (2017) 1650255

B31 (2017) 1750261

B31 (2017) 1750109

B31 (2017) 1750206

B31 (2017) 1744093

B31 (2017) 1750147

B31 (2017) 1750015

B31 (2017) 1750206

B31 (2017) 1750069

B31 (2017) 1750112

B31 (2017) 1750118

B31 (2017) 1750054

B31 (2017) 1750044

B31 (2017) 1750152

B31 (2017) 1750122

B31 (2017) 1750218

B31 (2017) 1750090

B31 (2017) 1740006 
ing in Bose-Einstein condensates

Nguenang, J.-P., see Djoufack, Z. I.

Nguyen, V. T., see Ha, V. T. $\mathrm{T}$.

Ni, H., see Mamat, A.

Ni, H., see Qi, Y.

$\mathrm{Ni}$, J., see Ma, J.

$\mathrm{Ni}$, Y., see Chen, Q.-M.

Nickabadi, S., see Rouhi, S.

Nie, L., see Chen, R.

Nikkar, A., see Ansari, R.

Nilima, N., Maisnam, M. \& Phanjoubam, S., Structural and magnetic properties of nanostructured $\mathrm{Li}-\mathrm{Ni}$-Co ferrite prepared by sol-gel method

Ning, L., see Qin, J.

Ning, S.-Y., see Li, Z.

Ning, W., see Wang, L.

Nishinari, K., see Tannai, T.

Niu, Z., see Dong, J.

Novoselov, A. \& Pavlovsky, O., Critical charge in gapped graphene: The role of screening of the interaction potential by $\sigma$-orbitals

Obada, A.-S. F., see Abdalla, M. S.

Ogino, A., see Shao, Z.

Okumuş, M., Investigation of thermal and optical properties of some quartet mixed hydrogen-bonded liquid crystals

Olajire, B. A. \& Musari, A. A., Thermodynamic and structural properties of liquid Al-Au alloys

Onate, C. A., see Onyeaju, M. C.

Öncan, M., Koç, F., Şahin, M. \& Köksal, K., The angular electronic band structure and free particle model of aromatic molecules: Highfrequency photon-induced ring current

Onyeaju, M. C., Ikot, A. N., Onate, C. A., Aghemenloh, E. \& Hassanabadi,
B31 (2017) 1750150

B31 (2017) 1750140

B31 (2017) 1750141

B31 (2017) 1745002

B31 (2017) 1745022

B31 (2017) 1744077

B31 (2017) 1744086

B31 (2017) 1750262

B31 (2017) 1750259

B31 (2017) 1750018

B31 (2017) 1750083

B31 (2017) 1750149

B31 (2017) 1750217

B31 (2017) 1750254

B31 (2017) 1750159

B31 (2017) 1744049

B31 (2017) 1750068

B31 (2017) 1750211

B31 (2017) 1744093

B31 (2017) 1750224

B31 (2017) 1750134

B31 (2017) 1750119

\section{B31 (2017) 1750095}

H., Electronic states in core/shell $\mathrm{GaN} / Y_{x} G a_{1-x} N$ quantum well (qw) with the modified PöschlTeller plus Woods-Saxon potential in the presence of electric field

B31 (2017) 1750119

B31 (2017) 1630016

Ortix, C., see Chang, C.-H.

Osipov, V. A., see Kolesnikov, D. V.

Osman, A.-N. A., see AbdelRady, A. S.

Ostoja-Starzewski, M., see Malyarenko, A.

Ould Baba, A., see Bamaarouf, $\mathrm{O}$.

Ouyang, Q., see Chen, B.

Paganelli, S. \& Apollaro, T. J. G., Irreversible work versus fidelity susceptibility for infinitesimal quenches

Pakniat, R., see Soltani, M.

Pan, H., see $\mathrm{Pu}$, J.

Pan, J., see Sun, Z

Pan, S., Mandal, A., Md. Sohel, A., Saha, A. K., Das, D. \& Gupta, A. S., High energy oxygen irradiation-induced defects in fe-doped semi-insulating indium phosphide by positron annihilation technique

Pan, X. \& Tu, D., Research on the effect of coverage rate on the surface quality in laser direct writing process

Pan, Y., Chen, S. \& Lin, Y., Vacancy-induced elastic properties and hardness of $\mathrm{CrB}_{4}$ : $A$ DFT calculation

Panda, A., Herojit Singh, L., Govindaraj, R., Abhaya, S., Yadav, R. K., Rajagopalan, S., Venugopal Rao, G., Ragunathan, V. \& Amarendra, G., Defect-induced magnetism in liquid sodiumexposed stainless steel as studied by Mössbauer and positron annihilation
B31 (2017) 1750124

B31 (2017) 1750091

B31 (2017) 1750236

B31 (2017) 1750182

B31 (2017) 1744101

B31 (2017) 1750065

B31 (2017) 1750198

B31 (2017) 1744044

B31 (2017) 1750240

B31 (2017) 1750019

B31 (2017) 1744018

B31 (2017) 1750096 
spectroscopic techniques Pandey, P. C., see Dixit, A.

Panwar, M., Sharma, S. K. \& Panwar, S., Temperature dependence of elastic constants using thermal energy for geophysical minerals

Panwar, S., see Panwar, M. Parvin, F., see Alam, M. A.

Pavlovsky, O., see Novoselov, A.

Peng, B., see Peng, P.

Peng, B., see Zhou, W.

Peng, H., Qi, W., Ji, W., Li, S. \& He, J., Structural stability of alloyed and core-shell cu-pt bimetallic nanoparticles

Peng, H., see Ding, Q.

Peng, J. F., Jin, X., Xu, Z. B., Cai, Z. B., Zhang, X. Y. \& Zhu, M. H., Study on bending fretting $\mathrm{fa}$ tigue damage in $17 \mathrm{CrN}$ iMo6 steel

Peng, P., Wu, Z., Yu, H., Qu, H., Peng, B. \& He, X., A study of partial layout of adhesive on the thermal drift of mems capacitive accelerometers

Peng, P., see Wu, J.

Peng, T., Yang, S., Xiang, F., Liang, Y., Li, Q., Gao, X. \& Liu, S., Film tension of liquid nano-film from molecular modeling

Peng, X., see Liao, L.

Peng, Y., see Guo, S.

Pennini, F., see Ferri, G. L.

Pergament, A., see Velichko, A.

Perminov, V., see Velichko, A.

Peschansky, V. G., see Galbova, O.

Petrucci, A., see Cardone, F. Phanjoubam, S., see Nilima, N.

Philominathan, P., see Deepa, B.

Piskunov, S., see Sokolov, M.

Plastino, A., see Ferri, G. L.
B31 (2017) 1750033

B31 (2017) 1750047

B31 (2017) 1750103

B31 (2017) 1750103

B31 (2017) 1750135

B31 (2017) 1750068

B31 (2017) 1741006

B31 (2017) 1750242

B31 (2017) 1741012

B31 (2017) 1744033

B31 (2017) 1744020

B31 (2017) 1741006 B31 (2017) 1750064

B31 (2017) 1750016 B31 (2017) 1750067

B31 (2017) 1750169

B31 (2017) 1750151

B31 (2017) 1650261

B31 (2017) 1650261

B31 (2017) 1750114

B31 (2017) 1750168

B31 (2017) 1750083

B31 (2017) 1750200

B31 (2017) 1750251

B31 (2017) 1750151
Poostforush, A., see Bordbar, G. H.

Przedborski, M., Sen, S. \& Harroun, T. A., Longterm behavior of hertzian chains between fixed walls is really equilibrium

B31 (2017) 1750097

Pu, J., Wu, M. F. \& Pan, H., Numerical analysis on temperature field in single-wire flux-aided backing-submerged arc welding

Pu, J., Yu, S. \& Li, Y., Parameter optimization of flux-aided backingsubmerged arc welding by using taguchi method

Pushkarev, S. S., see Khusyainov, D. I.

Putrolaynen, V., see Velichko, A.

Qaid, S. A. S., see Faizan, M.

Qi, W., see Jia, Y.

Qi, W., see Peng, H.

Qi, Y., Liu, X., Ni, H., Zheng, M., Chen, L., Wang, L., Jiang, Y., Zhao, M. \& Gao, J., Rectifying characteristics and tunable magnetoresistance in heterojunctions of $\mathrm{La}_{0.9} \mathrm{Hf}_{0.1} \mathrm{MnO}_{3} / 0.05$ wt. \% $\mathrm{Nb}$-doped $\mathrm{SrTiO}_{3}$

Qi, Y., see Mamat, A.

Qian, H., see $\mathrm{Xu}, \mathrm{C}$.

Qian, N., see Lei, W. Y.

Qiao, D. H., see Lei, J.

Qiao, D., see Zhi, M.

Qin, J. \& Ning, L., Entropy evolution of $a$ damped harmonic oscillator driven by quasimonochromatic noise and external periodic force

Qin, L., Li, Q. \& Yuan, Y. J., A study of CoFeB magnetic yoke based on planar electromagnet

Qin, S., see Wang, Z.

Qin, X. M., Zhang, Q. F., Duan, X. Y., Wang, X. C., Jiang, Y. H., Zhou, R. \& Tan, J., On the crys-
B31 (2017) 1745022

B31 (2017) 1745002

B31 (2017) 1744059

B31 (2017) 1745021

B31 (2017) 1741010

B31 (2017) 1741005

B31 (2017) 1750149

B31 (2017) 1744073

B31 (2017) 1750196 
tallization kinetics of $z r-$ (co, ni)-al bulk metallic glasses

Qin, Y., see Su, F.

Qiu, S. Y., see Fu, Z. H.

Qiu, S., see Han, D.

Qiu, X., see Zhang, W.

Qu, H., see Peng, P.

Qu, H., see Zhou, W.

$\mathrm{Qu}, \mathrm{L}$., see Hou, Q.

Qu, L., see Huo, Q.

$\mathrm{Qu}, \mathrm{L}$., see Xu, Z.

Qu, L.-F., see Hou, Q.-Y.

Qu, Y., see Zhu, X.

Qu, Z., see Wang, B.

Rabani, H., see Shariati, A.

Rachadi, A., see Bamaarouf, O.

Rachadi, A., see Lazfi, S.

Ragunathan, V., see Panda, A.

Rajagopalan, S., see Panda, A.

Rajeswarapalanichamy, R., see Iyakutti, K.

Rakha, S. A., see Rauf, A.

Ramay, S. M., see Faizan, M.

Ramesh, K. V., see Venkatesh, D.

Rameshkumar, P., see Alexandar, A.

Ramzannezhad, A., see Bahari, A.

Rao, K. H., see Chaitanya Varma, M.

Rashidian, Z., Bayati, P. \& Lorestaniwiess, Z., Effects of rashba spin-orbit coupling on the conductance of graphene-based nanoribbons

Rasolian, G., see Khosravi, $\mathrm{H}$.

Rauf, A., Shah, S. S. A., Rakha, S. A., Gul, M., Ahmad, I., Ullah, Z., Shah, I. A. \& Naseem, S., Inducing the magnetic character in reduced graphene oxide through incorporation of $\mathrm{Fe}_{2} \mathrm{O}_{3}$ nanoparticles

Rawat, A. \& Upadhyay, T. C., Study of ferroelectric
B31 (2017) 1744012

B31 (2017) 1750238

B31 (2017) 1744048

B31 (2017) 1744040

B31 (2017) 1750005

B31 (2017) 1741006

B31 (2017) 1750242

B31 (2017) 1650240

B31 (2017) 1750247

B31 (2017) 1750008

B31 (2017) 1750107

B31 (2017) 1745008

B31 (2017) 1750139

B31 (2017) 1750199

B31 (2017) 1750182

B31 (2017) 1750228

B31 (2017) 1750033

B31 (2017) 1750033

B31 (2017) 1750257

B31 (2017) 1750118

B31 (2017) 1750253

B31 (2017) 1750153

B31 (2017) 1750174

B31 (2017) 1750015

B31 (2017) 1750063

B31 (2017) 1750043

B31 (2017) 1750109

B31 (2017) 1750118 lead monohydrogen phosphate type crystals

Ray, A., see Jani, M.

Raza, R., see Abbas, G.

Rehman, A., see Affan, H.

Reinhard, B. M., see Lerch, S.

Ren, D., see Jiao, M.

Ren, H., see Song, Y.

Ren, J. F., see Cai, L. L.

Ren, X., Dong, L., Wang, J. \& Wang, X., Titanium microgram weight low to $50 \mathrm{mg}$ and measurement based on exchange weighing method

Reshak, A. H., see Naseri, M.

Riaz, M., Zia, R. \& Saleemi, F., Synthesis and evaluation of factors affecting the in vitro bioactivity and antibacterial activity of bioactive glass ceramics

Ridolfi, F., see Cardone, F.

Rocca, M. C., see Ferri, G. L.

Rogatko, M. \& Wysokiński, K. I., Holographic superconductors in the presence of dark matter

Rosada, A., see Cardone, F.

Rosas, A. \& Lindenberg, K., Pulse propagation in granular chains: The binary collision approximation

Rosato, A., see Blackmore, D.

Rosetto, F., see Cardone, F.

Roshanzamir-Nikou, M. \& Goudarzi, H., Magnetization of disclinated graphene in nonuniform magnetic field

Rostami, A., see Jafari, H.

Rostami, G., see Jafari, H.

Rouhi, S., Ansari, R. \& Nickabadi, S., Modal analysis of double-walled carbon nanocones using the finite element method

Rouhi, S., see Ansari, R.

Rowlands, D. A., see Zhang,
B31 (2017) 1750260

B31 (2017) 1750175

B31 (2017) 1750193

B31 (2017) 1750191

B31 (2017) 1740002

B31 (2017) 1744090

B31 (2017) 1750243

B31 (2017) 1750072

B31 (2017) 1741003

B31 (2017) 1750044

B31 (2017) 1650246 B31 (2017) 1750168

B31 (2017) 1750151

B31 (2017) 1745019

B31 (2017) 1750168

B31 (2017) 1742016

B31 (2017) 1742004

B31 (2017) 1750168

B31 (2017) 1750013

B31 (2017) 1750002

B31 (2017) 1750002

B31 (2017) 1750262

B31 (2017) 1750018 
J.

Roy, S., Gord, J. R., Hua, J.-C. \& Gunaratne, G. H., Robust-mode analysis of hydrodynamic flows

Rozi, A., see Mamat, A.

Rustaee, N., Tavassoly, M. K. \& Daneshmand, R., Damping, field-field correlation and dipoledipole interaction effects on the entanglement and atomic inversion dynamics

Saadi, M. F., Shirkani, H. \& Golshan, M. M., Effects of optical phonon interaction on dynamical valley polarization in graphene

Sabra, W., see Aly, A. H.

Sadhukhan, B., Bandyopadhyay, S., Nayak, A. \& Mookerjee, A., Disorder induced lifetime effects in binary disordered systems: A first principles formalism and an application to disordered graphene

Sadykov, N. R. \& Aporoski, A. V., Majorana fermion wavefunctions in carbon nanotubes and carbynes

Sadykova, O. G., see Kolesnikov, D. V.

Saha, A. K., see Pan, S.

Şahin, M., see Öncan, M.

Sahoo, J., Shadangi, N. \& Nayak, P., Phonon response of some heavy fermion systems in $d y$ namic limit

Saib, S., see Bouarissa, N.

Saidu, A., see Alajerami, Y. S. M.

Sakthy Priya, S., see Alexandar, A.

Sala, V., see Cardone, F.

Salah, A., see Abdel-Rady, A. S.

Salas, P., Solís, M. A., Fortes, M. \& Sevilla, F. J., Thermodynamic properties of underdoped $\mathrm{YBa}_{2} \mathrm{Cu}_{3} \mathrm{O}_{6+x} \quad$ cuprates
B31 (2017) 1750032

B31 (2017) 1742007 B31 (2017) 1745002

B31 (2017) 1750006

B31 (2017) 1750001 B31 (2017) 1750123

B31 (2017) 1750218

B31 (2017) 1750017

B31 (2017) 1750124

B31 (2017) 1750019

B31 (2017) 1750095

B31 (2017) 1750090

B31 (2017) 1750232

B31 (2017) 1750171

B31 (2017) 1750174

B31 (2017) 1750168

B31 (2017) 1750091 for several doping values

Salcic, Z., see Chen, F.

Salcic, Z., see Zhan, X.

Saleemi, F., see Riaz, M.

Sandouqa, A. S., see AlMaaitah, A. F.

Sandoval-Villalbazo, A., see Mariscal-Sanchez, A.

Sanjinés, D., see Mamani, E.

Sankaranarayanan, R., see Muthuganesan, R.

Santoro, E., see Cardone, F.

Sanyal, D., see Luitel, H.

Saxena, A., see Christov, I. C.

Schmidt-Böcking, H., see Afaneh, F.

Sedighy, S. H., see Fazeli, M.

Seifi, M., see Nasehnia, F.

Sen, P., see Maity, A.

Sen, S., see Blackmore, D.

Sen, S., see Kashyap, R.

Sen, S., see Przedborski, M.

Sen, S., see Tiwari, M.

Sen, S., see Westley, A.

Senay, M., see Algin, A.

Sevilla, F. J., see Salas, P.

Shadangi, N., see Sahoo, J.

Shah, I. A., see Rauf, A.

Shah, S. S. A., see Rauf, A.

Shajari, D., see Bahari, A.

Shakil, M., see Ahmed, S.

Shan, B., Wang, J., Zhang, L., Deng, B. \& Wei, X., Fitting of adaptive neuron model to electrophysiological recordings using particle swarm optimization algorithm

Shanahan, L., see Westley, A.

Shang, Y., Limit of a nonpreferential attachment multitype network model

Shang, Y.-Q., Hong, Q., Ma, Y.-L., Wu, Y.-L., Zhang, Y. \& Chen, J., Study on sapphire microstructure processing technology based on wet etching

Shao, F. \& Li, K., A graph model for preventing railway accidents based on
B31 (2017) 1750100

B31 (2017) 1744052

B31 (2017) 1744051

B31 (2017) 1650246

B31 (2017) 1750202

B31 (2017) 1750143

B31 (2017) 1750116

B31 (2017) 1750166

B31 (2017) 1750168

B31 (2017) 1750227

B31 (2017) 1742008

B31 (2017) 1750215

B31 (2017) 1750059

B31 (2017) 1750054

B31 (2017) 1750077

B31 (2017) 1742004

B31 (2017) 1742014

B31 (2017) 1742011

B31 (2017) 1742012

B31 (2017) 1742002

B31 (2017) 1650247

B31 (2017) 1750100

B31 (2017) 1750090

B31 (2017) 1750118

B31 (2017) 1750118

B31 (2017) 1750015

B31 (2017) 1650243

B31 (2017) 1750023

B31 (2017) 1742002

B31 (2017) 1750026

B31 (2017) 1741004 
the maximal information coefficient

Shao, Z., Ogino, A. \& Nagatsu, M., Construction and surfacel interface behavior of biofunctional surface layer by microwave-excited Ar/ $\mathrm{H}_{2} \mathrm{O}$ plasma-induced polyethylene glycol polymerization

Shao, Z., Shi, C., Chen, J. \& Zhang, Y., Preparation of SnS thin films with gear-like sheet appearance by close-spaced vacuum thermal evaporation

Shariati, A., Rabani, H. \& Mardaani, M., Electronic conductance of a poly( $p$ phenylene)-like nanowire in the presence of thermal atomic vibrations

Sharma, B. S., see Sunil, K.

Sharma, C., see Banerjee, K.

Sharma, D., see Bhattacherjee, A. B.

Sharma, R. \& Indu, B. D., Thermal transport in layered structure of $\mathrm{YBa}_{2} \mathrm{Cu}_{3} \mathrm{O}_{7-\delta}$ superconductors

Sharma, S. K., see Panwar, M.

Shehzad, N., see Khan, I.

Shen, F.-F., Lu, D.-F., Zhu, H.-W., Ji, C.-Y. \& Shi, Q.-F., Tunable far-field acoustic imaging by twodimensional sonic crystal with concave incident surface

Shen, J.-M., see Zhu, Q.-L.

Shen, L., Chen, H., Che, X. \& Xu, L., Corrosionfatigue crack propagation of aluminum alloys for high-speed trains

Shen, L., see Chen, Y.

Shen, S., see $\mathrm{Hu}, \mathrm{F}$.

Shen, Z.-C., see Wang, Y.C.

Sheng, J., see Su, C.
B31 (2017) 1750010

B31 (2017) 1744093

B31 (2017) 1744054

B31 (2017) 1750199 B31 (2017) 1750108

B31 (2017) 1750160

B31 (2017) 1750062

B31 (2017) 1750225

B31 (2017) 1750103

B31 (2017) 1750148

B31 (2017) 1650259

B31 (2017) 1750136

B31 (2017) 1744009

B31 (2017) 1744036

B31 (2017) 1750121

B31 (2017) 1744067 B31 (2017) 1744045
Shi, C., see Shao, Z.

B31 (2017) 1744054

Shi, G., see Dai, G.

Shi, J., see Liao, L.

Shi, P.-M., Li, Q. \& Han, D.Y., Stochastic resonance and MFPT in an asymmetric bistable system driven by correlated multiplicative colored noise and additive white noise

Shi, Q.-F., see Shen, F.-F.

Shi, S.-P., Liu, Y.-L., Deng, B.-L., Zhang, C.-Y. \& Jiang, G., Density functional theory study of the geometrical and electronic structures of $G e_{n} V^{(0, \pm 1)}(n=1-9)$ clusters

Shi, W., see Dai, G.

Shi, Y., see Hu, F.

Shi, Z., see Chen, X.

Shirkani, H., see Saadi, M. F.

Shirzadi, B., see Yarmohammadi, $M$.

Shizuya, K., Directexchange duality of the Coulomb interaction and collective excitations in graphene in a magnetic field

Shokri, A., see Ghobadi, Z. K.

Siddiqui, S. A., see Bouarissa, N.

Singh, R. N., see Arafin, S.

Siyushev, P., see Gerhardt, I.

Sokolov, M., Eglitis, R. I., Piskunov, S. \& Yu. Zhukovskii, F., ab initio hybrid DFT calculations of $\mathrm{BaTiO}_{3}$ bulk and baoterminated (001) surface $F$-centers

Solaymani, S., see Khosravi, $\mathrm{H}$.

Soldatov, A. V., see Bogolubov, N. N.

Soltani, M., Tavassoly, M. K. \& Pakniat, R., The influence of excitation number of photon-added coherent state field on the entanglement swapping process
B31 (2017) 1744087

B31 (2017) 1750067

B31 (2017) 1750113

B31 (2017) 1650259

B31 (2017) 1750022

B31 (2017) 1744087

B31 (2017) 1750121

B31 (2017) 1750146

B31 (2017) 1750001

B31 (2017) 1750045

B31 (2017) 1750176

B31 (2017) 1750106

B31 (2017) 1750232

B31 (2017) 1750031

B31 (2017) 1740004

B31 (2017) 1750251

B31 (2017) 1750109

B31 (2017) 1850044

B31 (2017) 1750198 
Solís, M. A., see Salas, P. Son, N. M., see Tuyen, H. V. Song, A., Wang, X., Chen, T. \& Wan, L., Band structures in a twodimensional phononic crystal with rotational multiple scatterers

Song, A., see Bao, K.

Song, F., see Wei, J.

Song, H., see Gao, W.

Song, J., Long, X., Hao, Y., Zhu, J. \& Guo, Y., $a b$ initio calculations on structural and electronic transport properties of six-atom gan clusters

Song, K., see Chen, X.

Song, Q.-L., see An, N.

Song, Y., Ren, H. \& Lei, J., Collaborations between CpG sites in DNA methylation

Souza, T. X. R. \& Marsiglio, F., The possible role of van hove singularities in the high $T_{c}$ of superconducting $\mathrm{H}_{3} \mathrm{~S}$

Spera, G., see Cardone, F.

Srivastava, U. C., Dynamical study of debye temperature and combined density of states of $\mathrm{TiO}_{2}$

Starosvetsky, Y., Jayaprakash, K. R. \& Vakakis, A. F., Traveling and solitary waves in monodisperse and dimer granular chains

Stashans, A., see Marcillo, F.

Stepanenko, D. I., see Galbova, O.

Su, C., Zhou, J., Meng, X. \& Sheng, J., Comparison of warm laser shock peening and laser shock peening techniques in lengthening the fatigue life of welded joints made of aluminum alloy

Su, F., Deng, B., Li, H., Yang, S., Qin, Y., Wang, J. \& Liu, C., FPGA-based hardware simulation of
B31 (2017) 1750100
B31 (2017) 1750128

B31 (2017) 1750038

B31 (2017) 1750125

B31 (2017) 1750007

B31 (2017) 1750042

B31 (2017) 1750222

B31 (2017) 1750146

B31 (2017) 1750089

B31 (2017) 1750243

B31 (2017) 1745003

B31 (2017) 1750168

B31 (2017) 1750020

B31 (2017) 1742001

B31 (2017) 1750111

B31 (2017) 1750114

B31 (2017) 1744045 nonlinear autoregressive volterra model to reconstruct the single neuron spike pattern

Su, F., Dong, H., Jia, L., Tian, Z. \& Sun, X., Space-time correlation analysis of traffic flow on road network

$\mathrm{Su}, \mathrm{H}$., see Chen, Y.

$\mathrm{Su}, \mathrm{H}$., see Li, Z.

Su, L.-X., see Wang, Y.-C.

Sun, B., see Li, J.

Sun, D., see Zhang, F.

Sun, D.-B., see Zhang, C.$\mathrm{H}$.

Sun, G., see Tserkezis, C.

Sun, H., Wu, Z. \& Tian, Q., Fractal dimension method for exciton in cylindrical GaAs/Al $G a_{1-x} A s$ coreshell-cap nanowires

Sun, K., see Xu, L. F.

Sun, L., Gao, Y., Yoshida, K., Yano, T., Li, Y. \& Liu, Y., Structural, mechanical, thermal and electronic properties of novel ternary carbide $\mathrm{Al}_{4} \mathrm{Si}_{2} \mathrm{C}_{5}$ under high pressure by DFT calculation

Sun, Q., see Fan, K.

Sun, Q., see Sun, Y.

Sun, R. X., Zheng, J., Zhan, L. J., Huang, S. Y., Li, H. T. \& Deng, Z. G., Design and fabrication of $a$ hybrid maglev model employing PML and SML

Sun, S. P., Hu, Y. F., Li, X. P., Chen, Y., Wang, H. J., Yu, Y., Jiang, Y. \& Yi, D. Q., The effect of point defect on mechanical properties of $\mathrm{MoSi}_{2}$

Sun, S., see Zhou, W.

Sun, X., see Su, F.

Sun, Y., Sun, Y., Sun, Q. \& Xiao, X., Lightweight ceramsites prepared by the solid waste of semiconductor and textile dyeing industry

Sun, Y., see Li, W.

Sun, Y., see Sun, Y.
B31 (2017) 1744097

B31 (2017) 1750238

B31 (2017) 1750027

B31 (2017) 1744057

B31 (2017) 1750217

B31 (2017) 1744067

B31 (2017) 1741014

B31 (2017) 1750223

B31 (2017) 1750138

B31 (2017) 1740005

B31 (2017) 1750209

B31 (2017) 1750133

B31 (2017) 1750012

B31 (2017) 1750080

B31 (2017) 1744097

B31 (2017) 1745014

B31 (2017) 1744081

B31 (2017) 1750242

B31 (2017) 1750027

B31 (2017) 1750210

B31 (2017) 1744097 
Sun, Y., see Zhang, Y.

B31 (2017) 1744003

Sun, Z., Cong, S., Pan, J. \& Zheng, J., A study for bank effect on ship traffic in narrow water channels using cellular automata

Sunil, K. \& Sharma, B. S., Thermoelastic properties of the earth lower mantle

Surendran, P., see Alexandar, A.

Surya, V. J., see Iyakutti, K. Tachibana, K., see Furuta, T. Tahne, B. A. \& Naderi, A., SLD-MOSCNT: A new MOSCNT with steplinear doping profile in the source and drain regions

Tajik, F., Khorrami, M., Masoudi, A. A. \& Dezashibi, F. M., The effect of roughness and correlation on the casimir torque between two plates

Tamandani, S. \& Darvish, G., Charge transfer modeling in monolayer circular graphene quantum dots-ZnO nanowires system for application in photovoltaic devices

Tan, H., see Jia, P.

Tan, J., see Qin, X. M.

Tan, J.-G., see Guo, Y.-F. Tan, X.-H., Kui, M.-Q., Li, X.-P., Mao, Z.-L. \& Xiao, H., Permeability and porosity models of bi-fractal porous media

Tan, Y., see Hu, Y.

Tanatar, B., see Khan, A.

Tang, C., see He, J.

Tang, H., see Yang, R.

Tang, H., see Ye, Y.

Tang, J., see Fan, K.

Tang, J., see Li, J.

Tang, L., see Zhi, M.

Tang, S. L., see Li, Y. F.

Tang, Z.-K., see Wang, Y.C.

Tannai, T. \& Nishinari, K., Congestion relaxation due to density-dependent junction rules in TASEP network

Tao, Y., Gu, H. \& Ding, $\mathrm{X}$., Spatial coherence resonance and spatial pattern transition induced by the decrease of inhibitory effect in a neuronal network

B31 (2017) 1750108

B31 (2017) 1750174

B31 (2017) 1750257

B31 (2017) 1742006

B31 (2017) 1650242

B31 (2017) 1750258

B31 (2017) 1650253

B31 (2017) 1744099

B31 (2017) 1744012

B31 (2017) 1750264

B31 (2017) 1750219

B31 (2017) 1750181

B31 (2017) 1750066

B31 (2017) 1750156

B31 (2017) 1744058

B31 (2017) 1744100

B31 (2017) 1750080

B31 (2017) 1745006

B31 (2017) 1741005

B31 (2017) 1750161

B31 (2017) 1744067
Tarawneh, O., see Batle, J.

Tavassoly, M. K., see Rustaee, $\mathrm{N}$.

Tavassoly, M. K., see Soltani, M.

Tay, S. L., Van Vliet, J., Wang, Y., Hou, F., Chao, X. \& Gao, W., Effects of $\mathrm{tio}_{2}$ sol on the microstructure and properties of Au-Co coatings

Tay, S. L., see Bo, H.

Tay, S. L., see Hou, F.

Tay, S. L., see Wang, Y.

Tay, S. L., see Wang, Y.

Tchoffo, M., see Lionel, T. $\mathrm{K}$.

Tegafaw, T., Xu, W., Lee, S. H., Chae, K. S., Chang, Y. \& Lee, G. H., Production of nearly monodisperse $\mathrm{Fe}_{3} \mathrm{O}_{4}$ and $\mathrm{Fe} @ \mathrm{Fe}_{3} \mathrm{O}_{4}$ nanoparticles in aqueous medium and their surface modification for biomedical applications

Tian, C.-L., see Zhong, M.$\mathrm{M}$.

Tian, Q., see Sun, H.

Tian, Y. L., see Cai, L. L.

Tian, Z., see Su, F.

Tie, S. \& Zhang, S., Removal of free carbon and crystal structure change of amorphous silica fume by calcination

Tie, S., see Jiang, Z.

Tie, S., see Liu, X.

Tiwari, M., Mohan, T. R. K. \& Sen, S., Impact decimation using alignment of granular spheres

Tiwari, S., see Dixit, A.

Tohyama, T., see Li, Z.

Tomchuk, P. M. \& Butenko, D., Nonlocal effects in metallic nanoparticles:
B31 (2017) 1750159

B31 (2017) 1750179 B31 (2017) 1750206

B31 (2017) 1750006

B31 (2017) 1750198

B31 (2017) 1744028

B31 (2017) 1744023

B31 (2017) 1744029

B31 (2017) 1744022

B31 (2017) 1744025

B31 (2017) 1750046

B31 (2017) 1750014

B31 (2017) 1750131

B31 (2017) 1750209

B31 (2017) 1750072

B31 (2017) 1750027

B31 (2017) 1744083

B31 (2017) 1744085

B31 (2017) 1744084

B31 (2017) 1742012

B31 (2017) 1750047

B31 (2017) 1750217 
the kinetic approach outlook

Topcu, G., see Algin, A.

Torkashvand, M., see Golivand, $\mathrm{M}$. B.

Trindade, M. A. S., Laws of large numbers for $q$ dependent random variables and nonextensive statistical mechanics

Tronin, V. N., see Borman, V. D.

Trügler, A., Hohenester, U. \& García de Abajo, F. J., Plasmonics simulations including nonlocal effects using a boundary element method approach

Tserkezis, C., Yan, W., Hsieh, W., Sun, G., Khurgin, J. B., Wubs, M. \& Asger Mortensen, N., On the origin of nonlocal damping in plasmonic monomers and dimers

Tu, C., Xie, L. \& Du, $\mathrm{X}$., Investigations of the spin hamiltonian parameters for $\mathrm{VO}^{2+}$ ions in $\mathrm{KZnCLSO}_{4} \cdot 3 \mathrm{H}_{2} \mathrm{O}$ single crystals

Tu, C., see Wang, N.

Tu, D., see Pan, X.

Tuan, D. A., Tung, V. T., Chuong, T. V. \& Van Hong, L., Influence of sintering temperature on structure, microstructure and piezoelectric properties of doped BZT-BCT ceramics

Tung, V. T., see Tuan, D. A. Tuyen, H. V. \& Son, N. M., Luminescence properties and energy transfer of $\mathrm{Tb}^{3+}-\mathrm{Eu}^{3+}$ co-doped $\mathrm{Sr}_{3} \mathrm{~B}_{2} \mathrm{O}_{6}$ phosphors

Törmä, P., see Nečada, M.

Uğur, G., see Baaziz, H.

Uğur, Ş., see Baaziz, H.

Ullah, H., see Faizan, M.

Ullah, Z., see Rauf, A.

Upadhyay, T. C., see Rawat, A.

Vakakis, A. F., see Starosvet-
B31 (2017) 1750029 B31 (2017) 1650247

B31 (2017) 1750003

B31 (2017) 1750117

B31 (2017) 1750201

B31 (2017) 1740007

B31 (2017) 1740005

B31 (2017) 1650262

B31 (2017) 1750154

B31 (2017) 1744018

B31 (2017) 1650258 B31 (2017) 1650258

B31 (2017) 1750128

B31 (2017) 1740006

B31 (2017) 1750226

B31 (2017) 1750226

B31 (2017) 1750253

B31 (2017) 1750118

B31 (2017) 1750260 sky, Y.

B31 (2017) 1742001

Van Hong, L., see Tuan, D. A.

B31 (2017) 1650258

B31 (2017) 1744028

Van Vliet, J., see Tay, S. L.

Velichko, A., Belyaev, M., Putrolaynen, V., Pergament, A. \& Perminov, V., Switching dynamics of single and coupled $\mathrm{VO}_{2}$ based oscillators as elements of neural networks

Venkatesh, D. \& Ramesh, K. V., Structural, dielectric and impedance spectroscopic studies of $\mathrm{Ni}_{0.5} \mathrm{Zn}_{0.5-x} \mathrm{Li}_{x} \mathrm{Fe}_{2} \mathrm{O}_{4}$ nanocrystalline ferrites

Venugopal Rao, G., see Panda, A.

Villamagua, L., see Marcillo, F.

Vinko, J. D., see Dufour, J.

Vinko, J. D., see Geneste, J.F.

Wan Shamsuri, W. N., see Bulus, I.

Wan, L., see Bao, K.

Wan, L., see Song, A.

Wan, L., see Wang, F.

Wan, Y., Li, M., Xie, E., Xu, S., Huang, Y. \& Deng, W., Luminescent properties and first-principles calculations of $(\mathrm{Cr}, \mathrm{Ca}): Y A G$ crystals

Wan, Z., see Zhu, Z.

Wang, A. Y., see Zuo, X.

Wang, A.-H., see Zhao, L.M.

Wang, B., Qu, Z., Li, X. \& Ma, J., The Lévy noise-induced current reversal phenomenon for self-propelled particles in a two-dimensional potential

Wang, B., see Bai, Y.

Wang, C., Li, Q. \& Hu, B., Preparation and characterization of $\mathrm{ZnS}$ nanoparticles prepared by hydrothermal method

Wang, C., see Chen, Y.-C.

Wang, C., see Ma, J.

Wang, C., see Wang, Z.
B31 (2017) 1650261

B31 (2017) 1750153

B31 (2017) 1750033

B31 (2017) 1750111

B31 (2017) 1745007

B31 (2017) 1745009

B31 (2017) 1750101

B31 (2017) 1750125

B31 (2017) 1750038

B31 (2017) 1750049

B31 (2017) 1744070

B31 (2017) 1744035

B31 (2017) 1744032

B31 (2017) 1750025

B31 (2017) 1750139 B31 (2017) 1750036

B31 (2017) 1744055

B31 (2017) 1750144

B31 (2017) 1650251

B31 (2017) 1750196 
Wang, C., see Zhang, G. Wang, D., see Zhou, W.

Wang, F., Chen, T., Wang, X., Bao, K. \& Wan, L., A membrane-type acoustic metamaterial muffler

Wang, G., see Gao, W.

Wang, G., see Yang, J.-J.

Wang, H. J., see Sun, S. P.

Wang, H., see Lin, L.

Wang, H., see Li, H.-J.

Wang, H., see Xia, S.

Wang, H., see Yao, C.

Wang, H., see Ye, Y.

Wang, J. T. \& Fan, J. D., Quantum states in a nanotube quantum dot

Wang, J., see Ding, M.

Wang, J., see Gao, Y.

Wang, J., see Li, H.

Wang, J., see Ren, X.

Wang, J., see Shan, B.

Wang, J., see Su, F.

Wang, K., see Fu, D.

Wang, K., see Zhang, W.

Wang, L., Jian, Z. \& Ning, W., First-principles study of the phonon, mechanical and thermodynamic properties of B2-phase aly under high pressures

Wang, L., see Du, L.

Wang, L., see Fan, K.

Wang, L., see Guo, L.

Wang, L., see Qi, Y.

Wang, L., see Zhang, F.

Wang, L., see Zhao, W.

Wang, L., see Zheng, Z.

Wang, L.-Q., see Yan, X.-A.

Wang, M., see Jia, Y.

Wang, M., see Xia, S.

Wang, N., Xie, L. \& Tu, C., Theoretical explanation of spin-hamiltonian parameters and local structure for the orthorhombic $\mathrm{MnO}_{4}^{2-}$ clusters in $\mathrm{K}_{2} \mathrm{SO}_{4}: \mathrm{Mn}^{6+}$ crystal

Wang, P., Yang, L., Dai, B., Yang, Z., Guo, S. \& Zhu, J., Nanoplatelike tungsten trioxide (hydrate) films prepared by crystal-seed-assisted
B31 (2017) 1750180 B31 (2017) 1750242

B31 (2017) 1750049 B31 (2017) 1750042

B31 (2017) 1744056

B31 (2017) 1744081

B31 (2017) 1750231

B31 (2017) 1750041

B31 (2017) 1650252

B31 (2017) 1744082

B31 (2017) 1744100

B31 (2017) 1745023

B31 (2017) 1744096

B31 (2017) 1750021

B31 (2017) 1750187

B31 (2017) 1741003

B31 (2017) 1750023

B31 (2017) 1750238

B31 (2017) 1750172

B31 (2017) 1750005

B31 (2017) 1750254

B31 (2017) 1750165

B31 (2017) 1741011

B31 (2017) 1750252

B31 (2017) 1745022

B31 (2017) 1750223

B31 (2017) 1750212

B31 (2017) 1744015

B31 (2017) 1650245

B31 (2017) 1741001

B31 (2017) 1650252

B31 (2017) 1750154 hydrothermal reaction

Wang, Q. M., see Zuo, X.

Wang, Q., Chen, H., Zhu, Z. \& Cui, Y., Mechanical properties of a dissimilar aluminum alloy joint welded by hybrid lasermig welding

Wang, Q., Xie, H., Chen, Y. \& Liu, C., Effects of temperature and pressure on the nucleation and growth of silver clusters from supersaturated vapor: A molecular dynamics analysis

Wang, R. L., see Xu, L. F.

Wang, T., see Mamat, A.

Wang, W. J., see Fu, Z. H.

Wang, W., see An, J.

Wang, W., see Jiao, P.

Wang, W., see Liu, Y.

Wang, W., see Xu, C.

Wang, X. C., see Qin, X. M.

Wang, X. F., see Dong, H. M.

Wang, X. \& Lv, J., Modeling and simulation of $d y$ namics of a planar-motion rigid body with friction and surface contact

Wang, X., see Bao, K.

Wang, X., see Chen, W.

Wang, X., see He, Y.

Wang, X., see Liao, H.

Wang, X., see Meng, M.

Wang, X., see Ren, X.

Wang, X., see Song, A.

Wang, X., see Wang, F.

Wang, X., see Zhang, M.

Wang, X., see Zhang, M.

Wang, X.-C., see Zhang, J.

Wang, Y. \& Yu, Y.-B., Plasmon excitations in doped square-lattice atomic clusters

Wang, Y., Hu, B., Tay, S. L., Hou, F., Gao, W. \& Chen, W., The microstructure and mechanical properties of $\mathrm{Ni}$ $\mathrm{Co}-\mathrm{ZrO}_{2}$ nanocomposite coatings

Wang, Y., Hu, B., Tay, S. L., Hou, F., Gao, W.
B31 (2017) 1744072

B31 (2017) 1744032

B31 (2017) 1744037

B31 (2017) 1750057

B31 (2017) 1750133

B31 (2017) 1745002

B31 (2017) 1744048

B31 (2017) 1744034

B31 (2017) 1750102

B31 (2017) 1744041

B31 (2017) 1744059

B31 (2017) 1744012

B31 (2017) 1750098

B31 (2017) 1744021

B31 (2017) 1750125

B31 (2017) 1744031

B31 (2017) 1744005

B31 (2017) 1744006

B31 (2017) 1744008

B31 (2017) 1741003

B31 (2017) 1750038

B31 (2017) 1750049

B31 (2017) 1741009

B31 (2017) 1741009

B31 (2017) 1745020

B31 (2017) 1750233

B31 (2017) 1744022 
\& Xiong, C., The microstructure and properties of sol-enhanced $\mathrm{Sn}-\mathrm{TiO}_{2}$ nanocomposite coatings

Wang, Y., Zhang, Y. \& Gao, W., Effect of $\mathrm{Zr}$ on the microstructures and mechanical properties of as-extruded $\quad \mathrm{Mg}-2.3 \mathrm{Zn}-$ $0.18 Y-x \mathrm{Zr}$ alloys

Wang, Y., see Bo, H.

Wang, Y., see Chen, W.

Wang, Y., see Hou, F.

Wang, Y., see Liu, Y.

Wang, Y., see Li, X.

Wang, Y., see Tay, S. L.

Wang, Y., see Yuan, X.

Wang, Y., see Zhang, C.-H.

Wang, Y., see Zhang, Y.

Wang, Y., see Zhuo, F.

Wang, Y.-C., Su, L.-X., Zhao, Y., Liu, J.-F., Shen, Z.-C., Feng, Y.-H., Wu, T.-Z. \& Tang, Z.-K., Resonant raman scattering study of $\mathrm{Be}_{x} \mathrm{Zn}_{1-x} \mathrm{O}$ thin films grown on sapphire by molecular beam epitaxy

Wang, Z. B., see Meng, M. Wang, Z., Qin, S. \& Wang, C., Properties of singlelayer graphene with supercell doped by one defect only

Wang, Z., see Chi, Z.

Wang, Z., see Jia, Y.

Wang, Z.-Y., see Chen, L.

Wang, see Fan, K.

Wei, F., see Guo, Y.-F.

Wei, H., see Hu, M.

Wei, J. Y. T., see Zhang, C.

Wei, J. \& Song, F., Association equilibria for proteins interacted with crowders of short-range attraction in crowded environment

Wei, X., see Shan, B.

Wei, Z.-X., see Wu, J.

Weil, E., see Dong, J.

Wen, D., see Zhao, X.

Wen, J., see Liu, C. G.

Wen, Y., see $\mathrm{Xu}, \mathrm{C}$.

Westley, A., De Meglio,
B31 (2017) 1744025

B31 (2017) 1744001

B31 (2017) 1744023

B31 (2017) 1744031

B31 (2017) 1744029

B31 (2017) 1744041

B31 (2017) 1744010

B31 (2017) 1744028

B31 (2017) 1650244

B31 (2017) 1750138

B31 (2017) 1744003

B31 (2017) 1750076

B31 (2017) 1744067

B31 (2017) 1744008

B31 (2017) 1750196

B31 (2017) 1750070

B31 (2017) 1741001

B31 (2017) 1744063

B31 (2017) 1741007

B31 (2017) 1750264

B31 (2017) 1750192

B31 (2017) 1745010

B31 (2017) 1750007

B31 (2017) 1750023

B31 (2017) 1750064

B31 (2017) 1744049

B31 (2017) 1750183

B31 (2017) 1750184

B31 (2017) 1744059
N., Hager, R., Mok, J. W., Shanahan, L. \& Sen, S., Study of simple land battles using agent-based modeling: Strategy and emergent phenomena

Westley, A., see Kashyap, $\mathrm{R}$.

Windows-Yule, C. R. K., Do granular systems obey statistical mechanics? A review of recent work assessing the applicability of equilibrium theory to vibrationally excited granular media

Wong, C. C., see Chen, F.

Wong, C. C., see Zhan, X.

Wrachtrup, J., see Gerhardt, I.

Wu, C.-X. \& Zhang, P., Solitary wave solution to a class of higher-order viscous traffic flow model

Wu, F., see Ma, J.

Wu, F., see Zhang, G.

Wu, F., see Zhu, X.

Wu, H., see Blackmore, D.

Wu, J. H., see Gao, N.

Wu, J., Huang, W.-Q., Yang, K., Wei, Z.-X., Peng, P. \& Huang, G.-F., Electronic and optical properties of $\mathrm{Cr}$-, B-doped, and $(\mathrm{Cr}, \mathrm{B})$ codoped $\mathrm{SrTiO}_{3}$

Wu, J., see Božović, I.

Wu, M. F., see $\mathrm{Pu}$, J.

$\mathrm{Wu}, \mathrm{R}$, see Yang, $\mathrm{T}$.

$\mathrm{Wu}, \mathrm{T}$., see Zhu, X.

Wu, T.-Z., see Wang, Y.-C.

Wu, W., see Zhang, J.

Wu, X. S., see Zhang, W. C.

Wu, X., see Yao, C.

Wu, Y., see Zhang, M.

Wu, Y.-L., see Shang, Y.-Q.

Wu, Z., see Peng, P.

Wu, Z., see Sun, H.

Wubs, M., see Tserkezis, C.

Wysokiński, K. I., see Rogatko, $\mathrm{M}$.

Xi, B., see Guo, Y.-F.

Xi, N., Hang, Z., Liu, Y., Chen, H., Zhang, M. \& Gao, W., Oxidation behavior of $\mathrm{HVOF}$ -
B31 (2017) 1742002

B31 (2017) 1742014

B31 (2017) 1742010

B31 (2017) 1744052

B31 (2017) 1744051

B31 (2017) 1740004

B31 (2017) 1750099

B31 (2017) 1650251

B31 (2017) 1750180

B31 (2017) 1744079

B31 (2017) 1742004

B31 (2017) 1750011

B31 (2017) 1750064

B31 (2017) 1745005

B31 (2017) 1744044

B31 (2017) 1750028

B31 (2017) 1744079

B31 (2017) 1744067

B31 (2017) 1741013

B31 (2017) 1750051

B31 (2017) 1744082

B31 (2017) 1741009

B31 (2017) 1741004

B31 (2017) 1741006

B31 (2017) 1750209

B31 (2017) 1740005

B31 (2017) 1745019

B31 (2017) 1750264 
sprayed nanostructured and $\mathrm{CeO}_{2}$-modified $\mathrm{WC}$ 12 Co coatings

Xia, F., see Zuo, X.

Xia, S., Liu, L., Kong, Y., Wang, H. \& Wang, M., Theoretical research on bandgap of $\mathrm{H}$-saturated $G a_{1-x} A l_{x} N$ nanowires

Xia, S., see Diao, Y.

Xiang, F., see Peng, T.

Xiang, H., see Zhang, X.

Xiao, F., Chen, H., Miao, J. \& Du, J., Effect of oxidation time on the microstructure and properties of ceramic coatings prepared by microarc oxidation on 7A04 superhard aluminum alloy

Xiao, H. B., see Xu, L. F.

Xiao, H., see Tan, X.-H.

Xiao, J., Yuan, H., Chen, L., Xiong, C., Ma, J., Zhao, Y., Chai, J., Du, W. \& Zhu, X., Anode-supported $\mathrm{BaZr}_{0.8} \mathrm{Y}_{0.2} \mathrm{O}_{3-\delta}$ membranes by tape casting and suspension spraying

Xiao, J., see Chen, L.

Xiao, J., see Fu, Z. H.

Xiao, J., see Xiong, C.

Xiao, J., see Xu, W.-L.

Xiao, J., see Zhao, Y.

Xiao, L., see Hu, M.

Xiao, X., see Sun, Y.

Xie, E., see Wan, Y.

Xie, H., see Wang, Q.

Xie, L., see Jiang, D.

Xie, L., see Tu, C.

Xie, L., see Wang, N.

Xie, Y., see Ji, Y.

Xie, Z., see Ding, M.

Xiong, C., Chen, L., Du, W., Ma, J., Xiao, J. \& Zhu, $\mathrm{X}$., Improve the properties of $n-\mathrm{ZnO} / \mathrm{p}$-Si heterojunction by CuSCN buffer layer

Xiong, C., see Bo, $\mathrm{H}$.

Xiong, C., see $\mathrm{Du}, \mathrm{W}$.

Xiong, C., see Hou, F.

Xiong, C., see Ma, J.

Xiong, C., see Wang, Y.

Xiong, C., see Xiao, J.
B31 (2017) 1744024 B31 (2017) 1744032

B31 (2017) 1650252

B31 (2017) 1750084

B31 (2017) 1750016

B31 (2017) 1740003

B31 (2017) 1744026

B31 (2017) 1750133

B31 (2017) 1750219

B31 (2017) 1744062

B31 (2017) 1744063

B31 (2017) 1744048

B31 (2017) 1744076

B31 (2017) 1744053

B31 (2017) 1744098

B31 (2017) 1750192

B31 (2017) 1744097

B31 (2017) 1744070

B31 (2017) 1750057

B31 (2017) 1650264

B31 (2017) 1650262

B31 (2017) 1750154

B31 (2017) 1744042

B31 (2017) 1744096

B31 (2017) 1744076

B31 (2017) 1744023

B31 (2017) 1744065

B31 (2017) 1744029

B31 (2017) 1744077

B31 (2017) 1744025

B31 (2017) 1744062
Xiong, C., see Xu, W.-L.

Xiong, C., see Yang, J.-J.

Xiong, C., see Zhao, Y.

Xiong, D., see Cao, X.

Xiong, D., see Deng, W.

$\mathrm{Xu}, \mathrm{B}$., see Zhang, W.

$\mathrm{Xu}$, C., Qian, H., Yong, H., Huang, X., Wang, W., Wen, Y., Zhong, K., Zhou, Y. \& Lai, M., Hydrothermal synthesis of $\mathrm{BiVO}_{4} / \mathrm{Bi}_{2} \mathrm{MoO}_{6}$ composites with enhanced photocatalytic activity

$\mathrm{Xu}$, D.-D., see Li, Z.

Xu, G. B., see Yan, J. G.

$\mathrm{Xu}$, G., Li, R., Fu, Q., Ma, Y. \& Guo, L., New nonbinary quantum codes with larger distance constructed from $\mathrm{BCH}$ codes over $\mathbb{F}_{q^{2}}$

Xu, G., Li, R., Guo, L. \& Lü, L., New optimal asymmetric quantum codes constructed from constacyclic codes

Xu, H.-C., Jiang, Z.-Q. \& Zhou, W.-X., Immediate price impact of a stock and its warrant: Power-law or logarithmic model?

$\mathrm{Xu}$, J., see Zhuo, F.

$\mathrm{Xu}, \mathrm{J} .$, Oblique discord

Xu, L. F., Sun, K., Feng, X., Xiao, H. B., Wang, R. L. \& Yang, C. P., Abnormal capacitance-voltage behaviors of bismuth-doped $\mathrm{CaCu}_{3} \mathrm{Ti}_{4} \mathrm{O}_{12}$ ceramics

Xu, L. \& Bu, W., Mechanical and thermodynamic properties of alx $(x=n, p$, as) compounds

$\mathrm{Xu}, \mathrm{L}$. \& Yan, B., $\mathrm{Fe}-6.5 \%$ $\mathrm{Si} / \mathrm{SiO}_{2}$ powder cores prepared by spark plasma sintering: Magnetic properties and sintering mechanism

Xu, L., see Chen, Y.

$\mathrm{Xu}, \mathrm{L}$. , see Shen, L.

$\mathrm{Xu}, \mathrm{S}$., see Cao, X.

$\mathrm{Xu}, \mathrm{S}$., see Chen, Z.
B31 (2017) 1744011

B31 (2017) 1744057

B31 (2017) 1744009

B31 (2017) 1744053

B31 (2017) 1744056

B31 (2017) 1744098

B31 (2017) 1744071

B31 (2017) 1744068

B31 (2017) 1750005

B31 (2017) 1744059

B31 (2017) 1750217

B31 (2017) 1650263

B31 (2017) 1750034

B31 (2017) 1750030

B31 (2017) 1750048

B31 (2017) 1750076

B31 (2017) 1650256

B31 (2017) 1750133

B31 (2017) 1750167

B31 (2017) 1744071

B31 (2017) 1744069 
Xu, S., see Wan, Y.

$\mathrm{Xu}, \mathrm{S}$., see Yao, C.

$\mathrm{Xu}, \mathrm{W}$., see Tegafaw, T.

Xu, W.-L., Yuan, H., Xiao, J., Xiong, C. \& Zhu, X., Morphology, photophysics and optoelectronics of P3HT nanoparticles and $\mathrm{TiO}_{2}$ nanorods composite

$\mathrm{Xu}$, Y., see Zhang, L.

$\mathrm{Xu}, \mathrm{Z}$. B., see Peng, J. F.

Xu, Z., Hou, Q. \& Qu, L., Effects on the optical properties and conductivity of Ag-N co-doped $\mathrm{ZnO}$

$\mathrm{Xu}, \mathrm{Z}$., see Huo, Q.

Xue, J., see Zhu, Z.

Xue, S., see Chen, Y.

Ya. Kezerashvili, R., see Lomsadze, R. A.

Yadav, R. K., see Panda, A. Yamaguchi, Y., see Liu, C.

Yamusa, Y. A., see Bulus, I.

Yan, B., see Ji, Y.

Yan, B., see Xu, L.

Yan, B., see Yan, L.

Yan, B., see Zheng, Z.

Yan, G.-A., see Lu, H.

Yan, H., see Yao, C.

Yan, J. G., Chen, Z. J., Xu, G. B., Kuang, Z., Chen, T. H. \& Li, D. H., Firstprinciples study of electronic structure, chemical bonding and elastic properties for new superconductor $\mathrm{CaFeAs}_{2}$

Yan, L. \& Yan, B., Microstructure and magnetic properties of $\mathrm{Fe}-6.5 \mathrm{wt} \%$ $\mathrm{Si} / \mathrm{MnZn}\left(\mathrm{Fe}_{2} \mathrm{O}_{4}\right)_{2} \quad \mathrm{com}$ posites with core-shell structure prepared by spark plasma sintering

Yan, W., see Tserkezis, C.

Yan, X., see Zhao, J.

Yan, X.-A., Wang, L.-Q., Zhang, W.-W., Liu, Y.W. \& Liu, H.-C., Switching on or off the optical bistability based on the interaction of double dark resonances

Yang, C. P., see Xu, L. F.
B31 (2017) 1744070
B31 (2017) 1744004
B31 (2017) 1750014

B31 (2017) 1744053

B31 (2017) 1744007

B31 (2017) 1744020

B31 (2017) 1750008

B31 (2017) 1750247

B31 (2017) 1744035

B31 (2017) 1744057

B31 (2017) 1650257

B31 (2017) 1750033

B31 (2017) 1744094

B31 (2017) 1750101

B31 (2017) 1744042

B31 (2017) 1744011

B31 (2017) 1744013

B31 (2017) 1744015

B31 (2017) 1750190

B31 (2017) 1744082

B31 (2017) 1650263

B31 (2017) 1744013

B31 (2017) 1740005

B31 (2017) 1745011

B31 (2017) 1650245 B31 (2017) 1750133
Yang, D. Y., see Liu, C. G.

Yang, G., see Liao, H.

Yang, H., see Zhang, W.

Yang, J., see Du, W.

Yang, J.-J., Wang, G., Du, W.-H. \& Xiong, C., Structural and electrical properties of $\mathrm{ZnO} / \mathrm{Zn}_{0.85} \mathrm{Mg}_{0.15} \mathrm{O}$ thin film prepared by pulsed laser deposition

Yang, J.-X. \& Zhang, X.-D., A spectral method to detect community structure based on distance modularity matrix

Yang, J.-X., see Zheng, B.$\mathrm{X}$.

Yang, K., Jiao, M., Yu, Y., Zhu, X., Liu, R. \& Cao, J., Thermal buffering performance of composite phase change materials applied in low-temperature protective garments

Yang, K., Li, J., Bao, Y. \& Jiang, Y., Microstructure and wear property of $\mathrm{Fe}-$ Cr13-C hardfacing alloy reinforced by WC particles

Yang, K., see Jiao, M.

Yang, K., see Wu, J.

Yang, L., see Dai, B.

Yang, L., see Fan, K.

Yang, L., see Liu, P.

Yang, L., see Wang, P.

Yang, P., see Chen, X.

Yang, R. \& Tang, H., Synthesis and luminescence properties of red-emitting $\mathrm{K}_{2} \mathrm{MgSiO}_{4}: \mathrm{Eu}^{3+}$ phosphors

Yang, R., see Chen, W.

Yang, R., see Zhang, W.

Yang, S., see Li, Y.

Yang, S., see Peng, T.

Yang, S., see Su, F.

Yang, T., Liu, D., Ji, J., Chen, J., Yu, Y. \& Wu, R., First-principles calculations for transition phase, mechanical and thermodynamic properties of $\mathrm{ZnS}$ under extreme condition
B31 (2017) 1750184
B31 (2017) 1744006
B31 (2017) 1750005
B31 (2017) 1744065

B31 (2017) 1744056

B31 (2017) 1750129

B31 (2017) 1775001

B31 (2017) 1744102

B31 (2017) 1744030

B31 (2017) 1744090

B31 (2017) 1750064

B31 (2017) 1744075

B31 (2017) 1750080

B31 (2017) 1741002

B31 (2017) 1744072

B31 (2017) 1750110

B31 (2017) 1744058

B31 (2017) 1744016

B31 (2017) 1750005

B31 (2017) 1750004

B31 (2017) 1750016

B31 (2017) 1750238

B31 (2017) 1750028 
Yang, T., see Chen, W.

Yang, T., see Chen, Y.

Yang, W. M., Yuan, X. C. \& Guo, Y. X., Influence of zno doping on the properties of single domain ybco bulks fabricated by $R E+011$ tsig process

Yang, X., see Zhang, C.

Yang, Y., see Bai, Y.

Yang, Z., see Wang, P.

Yannopapas, V., An atomistic-electrodynamics theory for the optical response of periodic lattices of metallic nanoparticles in the quantum size regime

Yano, T., see Sun, L.

Yao, C., Gao, W., Ye, Y., Jiang, Y., Xu, S. \& Yuan, X., The formation of periodic microl nano structured on stainless steel by femtosecond laser irradiation

Yao, C., Li, Y., Li, Q., Lu, S., Wu, X., Jiang, Y., Gao, W., Yan, H., Wang, H. \& Ye, Y., Preparation of broadband absorption ceramic coatings by using plasma electrolytic deposition

Yao, C., see Ye, Y.

Yao, P., see Liu, Y.

Yao, Y., Yi, M. \& Hou, D., Coherence resonance induced by cross-correlated sine-wiener noises in the Fitzhugh-Nagumo neurons

Yarmohammadi, M., Kazzaz, H. A. \& Feali, M. S., Orbital magnetoelectronic heat capacity of hydrogenated graphene in the presence of dilute charged impurity

Yarmohammadi, M., Mirabbaszadeh, K. \& Shirzadi, B., The effects of rashba spin-orbit coupling and holstein phonons on thermodynamic properties of
B31 (2017) 1744016

B31 (2017) 1745018

B31 (2017) 1750079

B31 (2017) 1750036

B31 (2017) 1744072

B31 (2017) 1740001

B31 (2017) 1750012

B31 (2017) 1744004

B31 (2017) 1744082

B31 (2017) 1744100

B31 (2017) 1744091

B31 (2017) 1750204

B31 (2017) 1750053
B31 (2017) 1750045

bn-doped graphene
Ye, Y., Jia, B., Chen, J., Jiang, Y., Tang, H., Wang, H., Luan, X., Liao, W., Zhang, C. \& Yao, C., Laser cleaning of the contaminations on the surface of tire mould

Ye, Y., see Yao, C.

Ye, Y., see Yao, C.

Yen, N. V., see Ha, N. T. T.

Yi Min, see Zhao, Z.

Yi, D. Q., see Sun, S. P.

Yi, G., see Li, H.

Yi, M., see Yao, Y.

Yin, L. \& Jones, M. I., Synthesis and characterization of $\beta$-sialon powders from Si, halloysite clay and $A L N$ powders

Yin, L., see Luo, D.

Ying, Q., see Ma, X. S.

Yong, H., see $\mathrm{Xu}, \mathrm{C}$.

Yoshida, K., see Sun, L.

Yousefi, R., see Ghoreishi, S. $S$.

Yu, B., see Fan, K.

Yu, B., see Fan, K.

Yu, H., see Peng, P.

Yu, H., see Zhou, W.

Yu, L., see Gao, N.

$\mathrm{Yu}$, M. \& Hua, J., Effect of sic particle size on the microstructure and properties of cold-sprayed Al/SiC ${ }_{p}$ composite coating

B31 (2017) 1744027

Yu, M., see Jiao, M.

$\mathrm{Yu}, \mathrm{S}$., see $\mathrm{Pu}$, J.

Yu, W., see Jiao, P.

Yu, Y., see Sun, S. P.

Yu, Y., see Yang, K.

Yu, Y., see Yang, T.

Yu, Y.-B., see Wang, Y.

Yu. Shchurova, L., see Namiot, V. A.

Yu. Zhukovskii, F., see Sokolov, M.

B31 (2017) 1744080

B31 (2017) 1745012

B31 (2017) 1650260

B31 (2017) 1744059

B31 (2017) 1750012

B31 (2017) 1750056

B31 (2017) 1741007

B31 (2017) 1741011

B31 (2017) 1741006

B31 (2017) 1750242

B31 (2017) 1750011

B31 (2017) 1744090

B31 (2017) 1744046

B31 (2017) 1750102

B31 (2017) 1744081

B31 (2017) 1744102

B31 (2017) 1750028

B31 (2017) 1750233

B31 (2017) 1750069

B31 (2017) 1750251

B31 (2017) 1744062

B31 (2017) 1744053

Yuan, H., see Xu, W.-L.

Yuan, H.-C., see Chen, Q.M.

B31 (2017) 1744086

B31 (2017) 1744098

B31 (2017) 1750076 
Entropy, energy, and entanglement of localized states in bent triatomic molecules

Yuan, X. B., see Cai, L. L.

Yuan, X. C., see Yang, W. M.

Yuan, X., Lin, G. \& Wang, Y., Design of elliptic cylindrical thermal cloak with layered structure

Yuan, X., see Yao, C.

Yuan, X., see Zhang, W.

Yuan, Y. J., see Chen, D.

Yuan, Y. J., see Qin, L.

Yuan, Y., see Zulfiqar

Yuan, Z., see Zhang, J.

Yurtseven, H., see Enginer, $\mathrm{Y}$.

Zad, H. A. \& Movahhedian, H., Classical correlation and quantum entanglement in the mixedspin Ising-XY model with Dzyaloshinskii-Moriya interaction

Zafar, M., see Ahmed, S.

Zang, G., see Huang, A.

Zarei, S., see Ghobadi, Z. K.

Zeng, G., see Guo, Q.

Zeng, J., see Zhu, X.

Zeng, M., see Liao, H.

Zhan, L. J., see Sun, R. X.

Zhan, X., Chen, F., Salcic, Z., Wong, C. C. \& Gao, W., Microstructure and enhanced photoluminescence of $\mathrm{ZnO} / \mathrm{V}_{2} \mathrm{O}_{5}$ composite

Zhan, X., see Chen, B.

Zhan, X., see Chen, F.

Zhang, A. M., see Zhang, W. C.

Zhang, A., see Ding, Q.

Zhang, C., Hou, H., Zhao, Y., Yang, X. \& Guo, Y., First-principles study on structural, elastic and thermal properties of $\gamma$ TiAl and $\alpha_{2}-T_{3} A l$ phases in tial-based alloy under high pressure

Zhang, C., Zhang, H. \& Wei, J. Y. T., Phase transformation in ultra-
B31 (2017) 1750088

B31 (2017) 1750072

B31 (2017) 1745018

B31 (2017) 1650244

B31 (2017) 1744004

B31 (2017) 1744092

B31 (2017) 1744074

B31 (2017) 1744073

B31 (2017) 1750234

B31 (2017) 1745020

B31 (2017) 1750245

B31 (2017) 1750094

B31 (2017) 1650243

B31 (2017) 1750087

B31 (2017) 1750106

B31 (2017) 1745001

B31 (2017) 1745008

B31 (2017) 1744006

B31 (2017) 1745014

B31 (2017) 1744051

B31 (2017) 1744101

B31 (2017) 1744052

B31 (2017) 1750051

B31 (2017) 1744033

B31 (2017) 1750079 thin $\mathrm{Y}-\mathrm{Ba}-\mathrm{Cu}-\mathrm{O}$ films by high-pressure oxygen annealing

Zhang, C., see Ye, Y.

Zhang, C.-H., Wang, Y. \& Sun, D.-B., The molecular dynamics simulation on the mechanical properties of Ni glass with external pressure

Zhang, E., see Zhang, K.

Zhang, F., Li, A., Bu, Z., Wang, L., Sun, D., Du, X. \& Gu, D., Vibration modes interference in the mems resonant pressure sensor

Zhang, G., Wu, F., Wang, C. \& Ma, J., Synchronization behaviors of coupled systems composed of hidden attractors

Zhang, H., see Zhang, C.

Zhang, J., Ke, S. H. \& Rowlands, D. A., Firstprinciples study on the dielectric and transport properties of the $\mathrm{LiNbO}_{3}$ type $\mathrm{CdPbO}_{3}$

Zhang, J., Wu, W. \& Bi, L., An automatic approach and grip method of microparticle in $3 d$ space

Zhang, J., Yuan, Z., Zhao, J.-F., Li, W.-M., Jia, Y.T., Liu, Y., Deng, Z., Zhang, S.-J., Feng, S.M., Liu, Q.-Q., Wang, X.-C. \& Jin, C.-Q., The study of the structure stability of hexagonal $\mathrm{Bi}\left(\mathrm{PO}_{4}\right)\left(\mathrm{H}_{2} \mathrm{O}\right)_{0.67}$ under high pressure

Zhang, J., see Ding, $\mathrm{H}$.

Zhang, J., see Liu, C. G.

Zhang, J.-X., see Li, Z.

Zhang, K., Zhang, E., Chen, H. \& Zhang, S., Spincurvature interaction from curved Dirac equation: Application to single-wall carbon nanotubes

Zhang, K., see Hu, M.
B31 (2017) 1750138

B31 (2017) 1750022

B31 (2017) 1744032

B31 (2017) 1750120

B31 (2017) 1745010

B31 (2017) 1744100

B31 (2017) 1750223

B31 (2017) 1750180

B31 (2017) 1745010

B31 (2017) 1750032

B31 (2017) 1741013

B31 (2017) 1745020

B31 (2017) 1750058

B31 (2017) 1750184

B31 (2017) 1750217

B31 (2017) 1750120

B31 (2017) 1750192 
Author index

Zhang, K., see Zhang, X.

Zhang, L., Xu, Y. \& Li, X., First-principles study of intermetallic compounds In CrMnFeCoNiZr system high-entropy alloy

Zhang, L., see Shan, B.

Zhang, M., Gou, G., Hang, Z. \& Chen, H., Effect of stress concentration on the fatigue strength of A7N01S-T5 welded joints

Zhang, M., Wu, Y., Wang, X. \& Wang, X., Alltransparent graphenebased flexible pressure sensor array

Zhang, M., see Xi, N.

Zhang, M., see Zhang, X.

Zhang, P., see Du, L.

Zhang, P., see Fu, D.

Zhang, P., see Guo, Q.

Zhang, P., see Wu, C.-X.

Zhang, Q. F., see Qin, X. M.

Zhang, Q., see Bai, Y.

Zhang, Q.-M., see Liu, Y.

Zhang, R., see Jiang, J.

Zhang, R., see Zhang, W.

Zhang, S., see Tie, S.

Zhang, S., see Zhang, K.

Zhang, S.-J., see Zhang, J.

Zhang, W. C., Zhang, A. M., Zhang, X., Zhou, G. T. \& Wu, X. S., Mixed magnetic exchange interactions and ferromagnetic diffuse phase transition of la $a_{1-x} \mathrm{mno}_{3+\delta}$ manganites

Zhang, W. \& Yuan, X., Novel surface treatment for natural fiber composites

Zhang, W., Luo, H., Zhang, R., Lu, X., Xu, B., Wang, K., Yang, R., Liu, J., Yang, H. \& Qiu, X., Angular-dependent magnetic torque in ironpnictide $\mathrm{BaFe}_{2-x} \mathrm{Ni}_{x} \mathrm{As}_{2}$

Zhang, W., see Ding, M.

Zhang, W.-W., see Yan, X.A.

Zhang, X. Y., see Peng, J. F. Zhang, X., Chen, J., Zhang, K. \& Chen, H., The
B31 (2017) 1744039

B31 (2017) 1744007

B31 (2017) 1750023

B31 (2017) 1744047

B31 (2017) 1741009

B31 (2017) 1744024

B31 (2017) 1740003

B31 (2017) 1750165

B31 (2017) 1750172

B31 (2017) 1745001

B31 (2017) 1750099

B31 (2017) 1744012

B31 (2017) 1750036

B31 (2017) 1744091

B31 (2017) 1750186

B31 (2017) 1750005

B31 (2017) 1744083

B31 (2017) 1750120

B31 (2017) 1745020

B31 (2017) 1750051

B31 (2017) 1744092

B31 (2017) 1750005 B31 (2017) 1744096

B31 (2017) 1650245 B31 (2017) 1744020 softening effect of heattreated strengthened $\mathrm{Al}$ $\mathrm{Zn}-\mathrm{Mg}$ alloy in welding process

Zhang, X., Xiang, H., Zhang, M. \& Lu, G., Plasmonic resonances of nanoparticles from largescale quantum mechanical simulations

Zhang, X., see Zhang, W. C.

Zhang, X.-D., see Yang, J.$\mathrm{X}$.

Zhang, Y. \& Cheng, X., First-principles study of li decorated coronene graphene

Zhang, Y., Wang, Y., Feng, T., Sun, Y., Dong, J. \& Gao, W., Effect of pores on the micromechanics of plasma-sprayed $\mathrm{Cr}_{3} \mathrm{C}_{2}-\mathrm{NiCr}$ coating in the nanoindentation testing

Zhang, Y., see Chen, B.

Zhang, Y., see Dai, G.

Zhang, Y., see Lei, W. Y.

Zhang, Y., see Shang, Y.-Q.

Zhang, Y., see Shao, Z.

Zhang, Y., see Wang, Y.

Zhang, Z., see $\mathrm{Hu}, \mathrm{M}$.

Zhao, C., see Hou, Q.

Zhao, C., see Zhu, X.

Zhao, C.-W., see Hou, Q.-Y.

Zhao, D., see Li, J.

Zhao, D., see Li, W.

Zhao, G., see Ding, Q.

Zhao, J., Yan, X. \& Gu, Q.,

The zeeman-split superconductivity with rashba and dresselhaus spinorbit coupling

Zhao, J.-F., see Zhang, J.

Zhao, L.-M., Zhou, Y.-S. \& Wang, A.-H., Effect of field modulation on the quasi-phase-matching for second harmonic generation in a twodimensional nonlinear photonic crystal

Zhao, M., see Qi, Y.

Zhao, M., see Zhu, X.

Zhao, W., Wang, L. \& Fan,
B31 (2017) 1744039

B31 (2017) 1740003

B31 (2017) 1750051

B31 (2017) 1750129

B31 (2017) 1750216

B31 (2017) 1744003

B31 (2017) 1744101

B31 (2017) 1744087

B31 (2017) 1745021

B31 (2017) 1741004

B31 (2017) 1744054

B31 (2017) 1744001

B31 (2017) 1750009

B31 (2017) 1650240

B31 (2017) 1744079

B31 (2017) 1750107

B31 (2017) 1741014

B31 (2017) 1750210

B31 (2017) 1744033

B31 (2017) 1745011

B31 (2017) 1745020

B31 (2017) 1750025

B31 (2017) 1745022

B31 (2017) 1745008 
J., Theory and method for weak signal detection in engineering practice based on stochastic resonance

Zhao, X., Li, D. \& Wen, D., Characteristics research of pressure sensor based on nanopolysilicon thin films resistors

Zhao, X., see Li, X.

Zhao, Y., Du, W.-H., Chen, L., Xiao, J., Xiong, C. \& Yuan, H.-C., Photoelectrocatalytic degradation of Rhodamine $B$ using graphene and titanium dioxide composite catalyst

Zhao, Y., see Chen, L.

Zhao, Y., see Du, W.

Zhao, Y., see Wang, Y.-C.

Zhao, Y., see Xiao, J.

Zhao, Y., see Zhang, C.

Zhao, Z., Yi MinZhou, P., Huang, Y. \& Zhong, C., Photon-assisted transport through a 1D-dotgraphene similar to STM model device

Zheng, B.-X., Die, D., Li, Q.-Q., Dai, M.-L., Li, Z.Q. \& Yang, J.-X., Comment on "the ground-state structures of $A u_{10}^{-}, A u_{8} N i$ and A $u_{9}$ Ni clusters"

Zheng, F., see Bai, Y.

Zheng, H., Duan, J. \& Dai, J., Synthesis of formamidinium lead iodide perovskite bulk single crystal and its optical properties

Zheng, J., see Lei, W. Y.

Zheng, J., see Sun, R. X.

Zheng, J., see Sun, Z.

Zheng, M., see Qi, Y.

Zheng, Q. L., see Li, Y. F.

Zheng, Z., Wang, L. \& Yan, B., Effects of laser power on the microstructure and mechanical properties of $316 L$ stainless steel prepared by selective laser melting

Zhi, M. H., see Lei, J.
B31 (2017) 1750212

B31 (2017) 1750183

B31 (2017) 1744010

B31 (2017) 1744098

B31 (2017) 1744063

B31 (2017) 1744065

B31 (2017) 1744067

B31 (2017) 1744062

B31 (2017) 1750079

\section{B31 (2017) 1750142}

B31 (2017) 1775001

B31 (2017) 1750036

B31 (2017) 1744066

B31 (2017) 1745021

B31 (2017) 1745014

B31 (2017) 1750240

B31 (2017) 1745022

B31 (2017) 1750161

B31 (2017) 1744015

B31 (2017) 1741010
Zhi, M., Tang, L. \& Qiao, D., Phase noise analysis of voltage controlled oscillator used in cesium atomic clock

Zhong, C., see Zhao, Z.

Zhong, K., see Xu, C.

Zhong, M.-M., Huang, C. \& Tian, C.-L., Phase stability, debye temperature and hardness of semiconducting manganese tetraboride $\mathrm{MnB}_{4}$ : Firstprinciples investigations

Zhong, S., see Lin, L.

Zhou, F.-F., see Chen, Q.-M.

Zhou, G. T., see Zhang, W. C.

Zhou, H., see Fu, D.

Zhou, H., see Li, H.

Zhou, J., see Su, C.

Zhou, P., see Zhao, Z.

Zhou, Q., see He, Y.

Zhou, R., see Qin, X. M.

Zhou, S., Influence of face-down and face-up bonding on the degree of polarization of superluminescent diode

Zhou, W., Li, F., Yu, H., Qu, H., Hao, R., Wang, D., Sun, S. \& Peng, B., Influence of adhesive nonuniformity on zero offset of micro accelerometer

Zhou, W.-X., see Xu, H.-C. Zhou, Y. \& Chen, L., Designing a new threedimensional periodic cellular auxetic material

Zhou, Y., see Xu, C.

Zhou, Y.-L., see Chen, Y.-C. Zhou, Y.-S., see Zhao, L.-M. Zhu, B., see Abbas, G.

Zhu, H.-W., see Shen, F.-F.

Zhu, J., see Dai, B.

Zhu, J., see Song, J.

Zhu, J., see Wang, P.

Zhu, L., see Bai, Y.

Zhu, M. H., see Peng, J. F.

Zhu, M., see Chen, Y.

Zhu, Q.-L., Hua, L. \& Shen, J.-M., Theoretical construction of weak topological crystalline insulators
B31 (2017) 1741005

B31 (2017) 1750142

B31 (2017) 1744059

B31 (2017) 1750131

B31 (2017) 1750231

B31 (2017) 1744086

B31 (2017) 1750051

B31 (2017) 1750172

B31 (2017) 1750187

B31 (2017) 1744045

B31 (2017) 1750142

B31 (2017) 1744005

B31 (2017) 1744012

B31 (2017) 1750229

B31 (2017) 1750242 B31 (2017) 1750048

B31 (2017) 1744088

B31 (2017) 1744059

B31 (2017) 1750144

B31 (2017) 1750025

B31 (2017) 1750193

B31 (2017) 1650259

B31 (2017) 1744075

B31 (2017) 1750222

B31 (2017) 1744072

B31 (2017) 1750036

B31 (2017) 1744020

B31 (2017) 1744036

B31 (2017) 1750136 
Zhu, S., see Ji, Y.

Zhu, X., Wu, F., Wu, T. \& Zhao, C., Remote sensing imaging simulation and cloud removal

Zhu, X., Zhao, M., Gao, J., Qu, Y., Zeng, J. \& Huang, W., Field effects on heterojunctions composed with electron-doped $\mathrm{La}_{1-x} \mathrm{Hf}_{x} \mathrm{MnO}_{3}$ and $\mathrm{Nb}$ $\mathrm{SrTiO}_{3}$ perovskites

Zhu, X., see Du, W.

Zhu, X., see Ma, J.

Zhu, X., see Xiao, J.

Zhu, X., see Xiong, C.

Zhu, X., see Xu, W.-L.

Zhu, X., see Yang, K.

Zhu, X.-F., see Chen, Q.-M.

Zhu, Y., see Fan, K.

Zhu, Y., see Fan, K.

Zhu, Y., see Hu, F.

Zhu, Z., Wan, Z., Li, Y., Xue, J. \& Hui, C., Intermediate layer, microstructure and mechanical properties of aluminum alloy/stainless steel butt joint using laser-MIG hybrid
B31 (2017) 1744042

B31 (2017) 1744079

B31 (2017) 1745008

B31 (2017) 1744065

B31 (2017) 1744077

B31 (2017) 1744062

B31 (2017) 1744076

B31 (2017) 1744053

B31 (2017) 1744102

B31 (2017) 1744086

B31 (2017) 1741007

B31 (2017) 1741011

B31 (2017) 1750121 welding-brazing method Zhu, Z., see Wang, Q.

Zhuo, F., Wang, Y., Fu, Y., Yuan, J. \& Xu, J., Synthesis and magnetic property of Eu-doped barium vanadate microflowers

Zia, R., see Riaz, M.

Ziegler, K., Controlling $d y$ namical entanglement in a josephson tunneling junction

Zilani, M. A. K., see Alam, M. A.

Zou, J., see Li, H.

$\mathrm{Zu}, \mathrm{X}$., see Fan, K.

Zulfiqar Yuan, Y., Iqbal, Z. \& Lu, J., Zn-doped $\mathrm{SnO}_{2}$ nanoparticles: Structural, optical, dielectric and magnetic properties

Zuo, X., Xia, F., Zhang, D., Ke, P. L., Wang, Q. M. \& Wang, A. Y., The effect of substrate bias on the characteristics of $\mathrm{CrN}$ coatings deposited by $D C$ superimposed HiPIMS system
B31 (2017) 1744035

B31 (2017) 1744037

B31 (2017) 1750076

B31 (2017) 1650246

B31 (2017) 1750255

B31 (2017) 1750135

B31 (2017) 1744043

B31 (2017) 1750080

B31 (2017) 1750234

B31 (2017) 1744032 